\title{
THE CANONICAL RAMSEY THEOREM AND COMPUTABILITY THEORY
}

\author{
JOSEPH R. MILETI
}

\begin{abstract}
Using the tools of computability theory and reverse mathematics, we study the complexity of two partition theorems, the Canonical Ramsey Theorem of Erdös and Rado, and the Regressive Function Theorem of Kanamori and McAloon. Our main aim is to analyze the complexity of the solutions to computable instances of these problems in terms of the Turing degrees and the arithmetical hierarchy. We succeed in giving a sharp characterization for the Canonical Ramsey Theorem for exponent 2 and for the Regressive Function Theorem for all exponents. These results rely heavily on a new, purely inductive, proof of the Canonical Ramsey Theorem. This study also unearths some interesting relationships between these two partition theorems, Ramsey's Theorem, and König's Lemma.
\end{abstract}

\section{INTRODUCTION}

König's Lemma and Ramsey's Theorem stand out as two of the most important and far-reaching results in countable combinatorics. There has been an extensive study of the strength of these combinatorial principles using the tools of computability theory and reverse mathematics. From the viewpoint of computability theory (see [21] for the necessary background information about computability theory), one may ask where solutions to computable instances of these problems lie either in the Turing degrees or the arithmetical hierarchy. Also, one may seek to classify the strength of these statements with respect to the reverse mathematics hierarchy (see [20] for the necessary background information about reverse mathematics). In this paper, we analyze the effective content of the Canonical Ramsey Theorem and the Regressive Function Theorem, and relate it to the effective content of König's Lemma and Ramsey's Theorem.

We list here some notational conventions. We denote the set of natural numbers by $\omega$. We identify each $n \in \omega$ with the set of elements less than it, so $n=\{0,1,2, \ldots, n-1\}$. Lowercase roman letters near the beginning or middle of the alphabet $(a, b, c, i, j, k, \ldots)$ will denote elements of $\omega$ (and sometimes -1 ), and lowercase roman letters near the end of the alphabet $(x, y, z, u, \ldots)$ will denote finite subsets of $\omega$. We identify a finite subset $x$ of $\omega$ of size $n$ with the $n$-tuple

Received by the editors August 29, 2005.

2000 Mathematics Subject Classification. Primary 03D80, 05D10.

Key words and phrases. Computability theory, Ramsey theory, recursion theory, reverse mathematics.

Most of the results in this paper appear in the author's dissertation written at the University of Illinois at Urbana-Champaign under the direction of Carl Jockusch with partial financial support provided by NSF Grant DMS-9983160. 
listing $x$ in increasing order and with the corresponding function $g: n \rightarrow \omega$. Uppercase roman letters near the end of the alphabet $(X, Y, Z, \ldots)$ will denote subsets of $\omega$, and uppercase roman letters near the beginning or middle of the alphabet $(A, B, C, H, I, J, \ldots)$ will denote infinite subsets of $\omega$. Given $X \subseteq \omega$, we denote the set of finite sequences of elements of $X$ by $X^{<\omega}$. We use $\sigma, \tau, \ldots$ to denote elements of $\omega^{<\omega}$. For a set $X \subseteq \omega$, we let $\operatorname{deg}(X)$ denote the Turing degree of $X$.

\section{Definition 1.1.}

(1) A tree is a subset $T$ of $\omega^{<\omega}$ such that for all $\sigma \in T$, if $\tau \in \omega^{<\omega}$ and $\tau \subseteq \sigma$, then $\tau \in T$.

(2) If $T$ is a tree and $S \subseteq T$ is also a tree, we say that $S$ is a subtree of $T$.

(3) A tree $T$ is bounded if there exists $h: \omega \rightarrow \omega$ such that for all $\sigma \in T$ and $k \in \omega$ with $|\sigma|>k$, we have $\sigma(k) \leq h(k)$.

(4) A branch of a tree $T$ is a function $f: \omega \rightarrow \omega$ such that $f \uparrow n \in T$ for all $n \in \omega$.

Theorem 1.2 (König's Lemma). Every infinite bounded tree has a branch.

\section{Definition 1.3.}

(1) Given a set $Z \subseteq \omega$ and $n \in \omega$, we let $[Z]^{n}=\{x \subseteq Z:|x|=n\}$.

(2) Suppose that $n, p \geq 1, B \subseteq \omega$ is infinite, and $f:[B]^{n} \rightarrow p$. Such an $f$ is called a $p$-coloring of $[B]^{n}$ and $n$ is called the exponent. We say that a set $H \subseteq B$ is homogeneous for $f$ if $H$ is infinite and $f(x)=f(y)$ for all $x, y \in[H]^{n}$.

Theorem 1.4 (Ramsey's Theorem [16]). Suppose that $n, p \geq 1, B \subseteq \omega$ is infinite, and $f:[B]^{n} \rightarrow p$. There exists a set $H$ homogeneous for $f$.

König's Lemma and Ramsey's Theorem are intimately related, as several proofs of partition theorems in set theory (such as Ramsey's Theorem) utilize paths through trees, and vice-versa. In the realm of large cardinals, those cardinals on which the appropriate analogue of Ramsey's Theorem holds are exactly those on which the appropriate analogue of König's Lemma holds (see [11, Theorem 7.8]).

Our interest is in the effective content of mathematical theorems. For example, we may ask whether every computable $f:[\omega]^{2} \rightarrow 2$ must have a computable homogeneous set. If the answer is negative, we may wonder about the complexity of homogeneous sets for computable $f:[\omega]^{2} \rightarrow 2$ as measured using the tools of computability theory. We might expect that the above mentioned relationship between partition theorems and König's Lemma manifests itself in their corresponding computability-theoretic or reverse mathematical strengths.

Aside from Ramsey's Theorem, our focus in this discussion is on two partition theorems which allow infinitely many colors: the Canonical Ramsey Theorem of Erdös and Rado and the Regressive Function Theorem of Kanamori and McAloon. We first set up some notation that will be useful when discussing partition theorems.

\section{Definition 1.5.}

(1) If $x \subseteq \omega$ is finite and $a \in \omega$, we write $x<a$ if $a$ is greater than every element of $x$.

(2) Suppose that $n \geq 1, B \subseteq \omega$ is infinite, $f:[B]^{n+1} \rightarrow \omega, x \in[B]^{n}$, and $a \in B$. When we write $f(x, a)$, we implicitly assume that $x<a$, and we let $f(x, a)=f(x \cup\{a\})$. Also, if $n=1$ and $a, b \in B$, when we write $f(a, b)$, we implicitly assume that $a<b$, and we let $f(a, b)=f(\{a, b\})$. 
The first partition theorem is the Canonical Ramsey Theorem due to Erdös and Rado which considers arbitrary functions $f:[\omega]^{n} \rightarrow \omega$. Of course, we cannot expect to always have homogeneous sets, as witnessed by the following simple functions $f:[\omega]^{2} \rightarrow \omega$ (where $\langle\cdot\rangle$ is a fixed effective bijection from $\omega^{2}$ to $\omega$ ):

(1) $f(a, b)=a$,

(2) $f(a, b)=b$,

(3) $f(a, b)=\langle a, b\rangle$.

However, the Canonical Ramsey Theorem for exponent 2 says that given any $f:[\omega]^{2} \rightarrow \omega$, there exists an infinite set $C \subseteq \omega$ which either is homogeneous, or on which $f$ behaves like one of the above functions. Precisely, given any $f:[\omega]^{2} \rightarrow \omega$, there exists an infinite $C$ such that either:

(1) For all $a_{1}, b_{1}, a_{2}, b_{2} \in C$, we have $f\left(a_{1}, b_{1}\right)=f\left(a_{2}, b_{2}\right)$.

(2) For all $a_{1}, b_{1}, a_{2}, b_{2} \in C$, we have $f\left(a_{1}, b_{1}\right)=f\left(a_{2}, b_{2}\right) \leftrightarrow a_{1}=a_{2}$.

(3) For all $a_{1}, b_{1}, a_{2}, b_{2} \in C$, we have $f\left(a_{1}, b_{1}\right)=f\left(a_{2}, b_{2}\right) \leftrightarrow b_{1}=b_{2}$.

(4) For all $a_{1}, b_{1}, a_{2}, b_{2} \in C$, we have $f\left(a_{1}, b_{1}\right)=f\left(a_{2}, b_{2}\right) \leftrightarrow\left(a_{1}=a_{2}\right.$ and $\left.b_{1}=b_{2}\right)$.

In the general case of an $f:[B]^{n} \rightarrow \omega$, we get $2^{n}$ different possibilities.

Definition 1.6. Suppose that $n \geq 1, B \subseteq \omega$ is infinite, $f:[B]^{n} \rightarrow \omega$, and $u \subseteq n$. We say that a set $C \subseteq B$ is $u$-canonical for $f$ if $C$ is infinite and for all $x_{1}, x_{2} \in[C]^{n}$, we have $f\left(x_{1}\right)=f\left(x_{2}\right) \leftrightarrow x_{1} \uparrow u=x_{2} \uparrow u$. We say that a set $C$ is canonical for $f$ if there exists $u \subseteq n$ such that $C$ is $u$-canonical for $f$.

Theorem 1.7 (Canonical Ramsey Theorem [3]). Suppose that $n \geq 1, B \subseteq \omega$ is infinite, and $f:[B]^{n} \rightarrow \omega$. There exists a set $C$ canonical for $f$.

Ramsey's Theorem is an immediate consequence of the Canonical Ramsey Theorem.

Claim 1.8. Suppose that $n, p \geq 1, B \subseteq \omega$ is infinite, and $f:[B]^{n} \rightarrow p$. If $C \subseteq B$ is canonical for $f$, then $C$ is homogeneous for $f$.

Proof. Suppose that $C \subseteq B$ is $u$-canonical for $f$, where $u \subseteq n$. Suppose that there exists $i<n$ such that $i \in u$. Fix $x_{k} \in[C]^{n}$ for all $k \in \omega$ such that $x_{0}<x_{1}<x_{2}<$ $\ldots$ For any $j, k \in \omega$ with $j \neq k$, we have $x_{j} \uparrow u \neq x_{k} \uparrow u$; hence $f\left(x_{j}\right) \neq f\left(x_{k}\right)$. This contradicts the fact that $f\left(x_{k}\right)<p$ for each $k \in \omega$. It follows that there is no $i<n$ such that $i \in u$, so $u=\emptyset$. Therefore, $C$ is homogeneous for $f$.

Our other main interest is the Regressive Function Theorem. The primary interest in this partition theorem is that Kanamori and McAloon showed that its finitary version is true but not provable in Peano Arithmetic (see [12]). Paris and Harrington [14] provided the first such natural finitary combinatorial statement, but Kanamori and McAloon's is arguably more natural. Like the Canonical Ramsey Theorem, it deals with colorings which allow infinitely many colors, but it places a restriction on which such colorings it considers.

Definition 1.9. Suppose that $n \geq 1, B \subseteq \omega$ is infinite, and $f:[B]^{n} \rightarrow \omega$.

(1) We say that $f$ is regressive if for all $x \in[B]^{n}$, we have $f(x)<\min (x)$ whenever $\min (x)>0$, and $f(x)=0$ whenever $\min (x)=0$.

(2) We say that a set $M \subseteq B$ is minhomogeneous for $f$ if $M$ is infinite and for all $x, y \in[M]^{n}$ with $\min (x)=\min (y)$ we have $f(x)=f(y)$. 
Theorem 1.10 (Regressive Function Theorem [12]). Suppose that $n \geq 1, B \subseteq \omega$ is infinite, and $f:[B]^{n} \rightarrow \omega$ is regressive. There exists a set $M$ minhomogeneous for $f$.

The Regressive Function Theorem (for exponent $n$ ) is a straightforward consequence of the Canonical Ramsey Theorem (for exponent $n$ ).

Claim 1.11 (Kanamori and McAloon [12]). Suppose that $n \geq 1, B \subseteq \omega$ is infinite, and $f:[B]^{n} \rightarrow \omega$ is regressive. If $C \subseteq B$ is canonical for $f$, then $C$ is minhomogeneous for $f$.

Proof. If $n=1$, then every infinite subset of $B$ is minhomogeneous for $f$, so we may assume that $n \geq 2$. Suppose that $C \subseteq B$ is $u$-canonical for $f$, where $u \subseteq n$. Suppose that there exists $i$ with $0<i<n$ such that $i \in u$. Let $c_{0}=\min (C)$. Fix $x_{k} \in[C]^{n-1}$ for all $k \in \omega$ such that $c_{0}<x_{0}<x_{1}<x_{2}<\ldots$ For any $j, k \in \omega$ with $j \neq k$, we have $\left(c_{0}, x_{j}\right) \uparrow u \neq\left(c_{0}, x_{k}\right) \uparrow u$; hence $f\left(c_{0}, x_{j}\right) \neq f\left(c_{0}, x_{k}\right)$. This contradicts the fact that $f\left(c_{0}, x_{k}\right) \leq c_{0}$ for each $k \in \omega$. It follows that there is no $i$ with $0<i<n$ such that $i \in u$, so either $u=\emptyset$ or $u=\{0\}$. If $u=\emptyset$, then $C$ is homogeneous for $f$, and hence minhomogeneous for $f$. If $u=\{0\}$, then for all $x, y \in[C]^{n}$, we have $f(x)=f(y) \leftrightarrow x\lceil\{0\}=y\lceil\{0\} \leftrightarrow \min (x)=\min (y)$, so $C$ is minhomogeneous for $f$.

Before embarking on a study of the Canonical Ramsey Theorem and the Regressive Function Theorem, we will discuss some of the known results for König's Lemma and Ramsey's Theorem.

\section{Effective Analysis of KÖNig's Lemma And Ramsey's Theorem}

An effective analysis of König's Lemma depends on both the complexity of $f$ and the complexity of the bound. We will mostly be concerned with subtrees of $2^{<\omega}$ (that is, trees which are bounded by $h(k)=1$ ). It is straightforward to effectively code computable trees bounded by a computable function using computable subtrees of $2^{<\omega}$, so for our purposes there is no loss in restricting attention to the following case.

Corollary 2.1 (Weak König's Lemma). Every infinite subtree of $2^{<\omega}$ has a branch.

Definition 2.2. Let $\mathbf{a}$ and $\mathbf{b}$ be Turing degrees. We write $\mathbf{a} \gg \mathbf{b}$ to mean that every infinite b-computable subtree of $2^{<\omega}$ has an a-computable branch.

The notation $\mathbf{a} \gg \mathbf{b}$ was introduced in Simpson [19], and many of the basic properties of this ordering can be found there. It is well known that $\mathbf{a} \geq \mathbf{b}^{\prime} \rightarrow \mathbf{a} \gg$ $\mathbf{b} \rightarrow \mathbf{a}>\mathbf{b}$. The following proposition gives some equivalent characterizations of this ordering.

Proposition 2.3 (Scott [17], Solovay). Let $\mathbf{a}$ and $\mathbf{b}$ be Turing degrees. The following are equivalent:

(1) $\mathbf{a} \gg \mathbf{b}$.

(2) Every partial $\{0,1\}$-valued $\mathbf{b}$-computable function has a total a-computable extension.

(3) a is the degree of a complete extension of the theory of Peano Arithmetic with an additional unary predicate symbol $P$, axioms $P(n)$ for all $n \in B$ and $\neg P(n)$ for all $n \notin B$ (where $B$ is a fixed set in $\mathbf{b})$, and induction axioms for formulas involving $P$. 
Using the existence of a computable tree in which the branches code complete extensions of Peano Arithmetic, it follows that there is a "universal" computable subtree of $2^{<\omega}$.

Corollary 2.4 (Scott [17]). There exists an infinite computable subtree $T$ of $2^{<\omega}$ such that given any branch $B_{T}$ of $T$, and any infinite computable subtree $S$ of $2^{<\omega}$, there exists a branch $B_{S}$ of $S$ such that $B_{S} \leq_{T} B_{T}$.

In [10], Jockusch and Soare established the following fundamental result.

Theorem 2.5 (Low Basis Theorem [10, Theorem 2.1]). There exists $\mathbf{a} \gg \mathbf{0}$ with $\mathbf{a}^{\prime}=\mathbf{0}^{\prime}$.

We now turn to Ramsey's Theorem. Specker [22] was the first to analyze the effective content of Ramsey's Theorem, and he showed that there exists a computable $f:[\omega]^{2} \rightarrow 2$ with no computable homogeneous set. Before discussing further bounds on the complexity of homogeneous sets, we first examine a few proofs of Ramsey's Theorem.

Definition 2.6. Suppose that $n, p \geq 1, B \subseteq \omega$ is infinite, and $f:[B]^{n+1} \rightarrow p$. We say that a pair $(A, g)$, where $A \subseteq B$ is infinite and $g:[A]^{n} \rightarrow p$, is a prehomogeneous pair for $f$ if $f(x, a)=g(x)$ for all $x \in[A]^{n}$ and all $a \in A$.

Most proofs of Ramsey's Theorem break down into the following three steps and differ only in their proofs of (1):

(1) Given $f:[B]^{n+1} \rightarrow p$, construct a prehomogeneous pair $(A, g)$ for $f$.

(2) Apply induction to $g:[A]^{n} \rightarrow p$.

(3) Show that any set homogeneous for $g$ is homogeneous for $f$.

We first establish (3).

Claim 2.7. Suppose that $n, p \geq 1, B \subseteq \omega$ is infinite, $f:[B]^{n+1} \rightarrow p$, and $(A, g)$ is a prehomogeneous pair for $f$. If $H$ is homogeneous for $g$, then $H$ is homogeneous for $f$.

Proof. Let $x_{1}, x_{2} \in[H]^{n}$, let $a_{1}, a_{2} \in H$, and suppose that $x_{1}<a_{1}$ and $x_{2}<a_{2}$. We then have

$$
\begin{aligned}
f\left(x_{1}, a_{1}\right) & =g\left(x_{1}\right) & & (\text { since }(A, g) \text { is a prehomogeneous pair for } f) \\
& =g\left(x_{2}\right) & & (\text { since } H \text { is homogeneous for } g) \\
& =f\left(x_{2}, a_{2}\right) & & (\text { since }(A, g) \text { is a prehomogeneous pair for } f) .
\end{aligned}
$$

Therefore, $H$ is homogeneous for $f$.

The standard way to construct a prehomogeneous pair proceeds by repeatedly thinning down a set of candidates to add to the prehomogeneous pair, while ensuring that this set of candidates remains infinite. For simplicity, consider a function $f:[\omega]^{2} \rightarrow 2$. We will enumerate $A$ in increasing order as $a_{0}, a_{1}, \ldots$ We begin by letting $a_{0}=0$. If there are infinitely many $b \in \omega$ with $f\left(a_{0}, b\right)=0$, then we can define $g\left(a_{0}\right)=0$ and restrict attention to the set $I_{0}=\left\{b \in \omega: f\left(a_{0}, b\right)=\right.$ $0\}$. Otherwise, there are infinitely many $b \in \omega$ with $f\left(a_{0}, b\right)=1$, so we can define $g\left(a_{0}\right)=1$ and restrict attention to the set $I_{0}=\left\{b \in \omega: f\left(a_{0}, b\right)=1\right\}$. We then let $a_{1}=\min I_{0}$ and continue in this fashion. If we succeed infinitely many times in this manner with color 0 , then the corresponding elements form a homogeneous set colored 0 , while if we succeed infinitely many times with color 1 , 
then the corresponding elements form a homogeneous set colored 1. Notice that this decision (infinitely many colored 0 or infinitely many colored 1 ) amounts to finding a homogeneous set for $g:[A]^{1} \rightarrow 2$.

This general idea can be extended to higher exponents $n$ and to all $p \geq 1$. Suppose that $B$ is computable and that $f:[B]^{n} \rightarrow p$ is computable. A simple analysis of this proof shows that there exists a prehomogeneous pair $(A, g)$ for $f$ with $\operatorname{deg}(A \oplus g) \leq \mathbf{0}^{\prime \prime}$ because the questions that need to be answered are whether or not certain effectively given sets are infinite. Following this outline, one arrives at the following result.

Theorem 2.8. Suppose that $n, p \geq 1, B \subseteq \omega$ is infinite, and $f:[B]^{n} \rightarrow p$. Suppose also that $B$ and $f$ are computable. There exists a set $H$ homogeneous for $f$ such that $\operatorname{deg}(H) \leq \mathbf{0}^{(2 n-2)}$.

Another approach is to build a prehomogeneous pair by coding such pairs into the branches of a $\mathbf{0}^{\prime}$-computable subtree of $2^{<\omega}$. Using an argument along these lines gives the following result.

Proposition 2.9 (essentially Jockusch [9, Lemma 5.4]). Suppose that $n, p \geq 1$, $B \subseteq \omega$ is infinite, and $f:[B]^{n+1} \rightarrow p$. Suppose also that $B$ and $f$ are computable and $\mathbf{a} \gg \mathbf{0}^{\prime}$. There exists a prehomogeneous pair $(A, g)$ for $f$ with $\operatorname{deg}(A \oplus g) \leq \mathbf{a}$. In particular (by the Low Basis Theorem), there exists a prehomogeneous pair $(A, g)$ for $f$ with $\operatorname{deg}(A \oplus g)^{\prime} \leq \mathbf{0}^{\prime \prime}$.

Iterating this result, we conclude the following.

Theorem 2.10 (essentially Jockusch [9, Theorem 5.6]). Suppose that $n, p \geq 1$, $B \subseteq \omega$ is infinite, and $f:[B]^{n} \rightarrow p$. Suppose also that $B$ and $f$ are computable and $\mathbf{a} \gg \mathbf{0}^{(n-1)}$. There exists a set $H$ homogeneous for $f$ such that $\operatorname{deg}(H) \leq \mathbf{a}$.

Jockusch also characterized the location of homogeneous sets for computable colorings in the arithmetical hierarchy for all exponents.

Theorem 2.11 (Jockusch [9, Theorem 5.1, Theorem 5.5]).

(1) Suppose that $n, p \geq 1, B \subseteq \omega$ is infinite, and $f:[B]^{n} \rightarrow p$. Suppose also that $B$ and $f$ are computable. There exists a $\Pi_{n}^{0}$ set homogeneous for $f$.

(2) For each $n \geq 2$, there exists a computable $f:[\omega]^{n} \rightarrow 2$ such that no $\Sigma_{n}^{0}$ set is homogeneous for $f$.

Furthermore, Jockusch and Hummel showed that the halting problem can be coded into the homogeneous sets of a computable $f:[\omega]^{3} \rightarrow 2$ and a c.e. $f:[\omega]^{2} \rightarrow 2$. These results will play important roles in the coding techniques used below.

Proposition 2.12 (Jockusch [9, Lemma 5.9]). For every $n \geq 3$, there exists a computable $h:[\omega]^{n} \rightarrow 2$ such that for all sets $H$ homogeneous for $h$, we have $h\left([H]^{n}\right)=\{0\}$ and $H \geq_{T} 0^{(n-2)}$.

Proposition 2.13 (Jockusch and Hummel [6, Lemma 3.7]). There exists a computably enumerable $h:[\omega]^{2} \rightarrow 2$ (that is, $\left\{x \in[\omega]^{2}: h(x)=1\right\}$ is c.e.) such that for all sets $H$ homogeneous for $h$, we have $h\left([H]^{2}\right)=\{0\}$ and $H \geq_{T} 0^{\prime}$.

The above arguments can also be used to give results in reverse mathematics.

Definition 2.14. We let $\mathrm{RT}_{p}^{n}$ denote the statement, in second-order arithmetic, that every $f:[\mathbb{N}]^{n} \rightarrow p$ has a homogeneous set. 
Formalizing the proofs of Ramsey's Theorem and of Proposition 2.12 in secondorder arithmetic, we arrive at the following.

Corollary 2.15 (Simpson). Let $n \geq 3$ and $p \geq 2$. Over $\mathrm{RCA}_{0}, \mathrm{RT}_{p}^{n}$ is equivalent to $\mathrm{ACA}_{0}$.

At this point, we are still left with many questions about the degrees of homogeneous sets for computable colorings for exponent 2. For a computable $f:[\omega]^{2} \rightarrow 2$, we know that we can find homogeneous sets below any $\mathbf{a} \gg \mathbf{0}^{\prime}$, but we don't know if we can code anything nontrivial. Furthermore, this gap for exponent 2 propagates up to higher exponents. A major step toward resolving this question was taken by Seetapun, who showed that it was not possible to code nontrivial information into the homogeneous sets of a computable coloring of exponent 2 .

Theorem 2.16 (Seetapun [18]). Suppose that $p \geq 2, B \subseteq \omega$ is infinite, and $f:[B]^{2} \rightarrow p$. Suppose also that $B$ and $f$ are computable and that $\left\{\mathbf{d}_{k}\right\}_{k \in \omega}$ is a family of nonzero degrees. There exists a set $H$ homogeneous for $f$ such that $\mathbf{d}_{k} \not \leq \operatorname{deg}(H)$ for all $k \in \omega$.

Seetapun iterated his result to arrive at the following important reverse mathematical fact.

Corollary 2.17. For each $p \geq 2, \mathrm{RCA}_{0}+\mathrm{RT}_{p}^{2}$ does not imply $\mathrm{ACA}_{0}$.

Adding the formal statements of Weak König's Lemma in second-order arithmetic to the base axiom system $\mathrm{RCA}_{0}$ of reverse mathematics gives the important system $\mathrm{WKL}_{0}$. Using the Low Basis Theorem and Theorem 2.11, one can show that for each $p \geq 2, \mathrm{RT}_{p}^{2}$ is not provable from $\mathrm{WKL}_{0}$ (a result first proved by Hirst [5, Corollary 6.9]); hence $\mathrm{RT}_{p}^{2}$ is not equivalent to any of the standard systems of reverse mathematics.

To get more information about the complexity of Ramsey's Theorem for exponent 2, we look for guidance from yet another proof of Ramsey's Theorem. Another proof of Ramsey's Theorem which is quite similar to the outline above uses a nonprincipal ultrafilter on $\omega$ (containing $B$ as an element) to guide the inductive construction. This changes the argument in the following fundamental manner. In the above outline, the key question is how to define $g\left(a_{n}\right)$ so that the corresponding thinned out set remains infinite. We know that some choice will succeed, but there may be many possible choices which work. In contrast, the ultrafilter guides us because exactly one of the corresponding sets will remain in the ultrafilter. In our context of effectively analyzing these proofs, the nonprincipal ultrafilter can be replaced by a more basic object.

Definition 2.18. A set $V \subseteq \omega$ is $r$-cohesive if $V$ is infinite and for every computable set $Z$, either $V \cap Z$ is finite or $V \cap \bar{Z}$ is finite.

Notice that if $V$ is an r-cohesive set, then $\left\{Z \subseteq \omega: Z\right.$ is computable and $\left.V \subseteq \subseteq^{*} Z\right\}$ is a nonprincipal ultrafilter in the Boolean algebra of computable sets. Hence, if $B$ is computable and $f:[B]^{n} \rightarrow p$ is computable, we can use an r-cohesive set in place of a nonprincipal ultrafilter on $\omega$ in the above construction. Jockusch and Stephan [7] (see also [8] for a correction) characterized the Turing degrees of jumps of r-cohesive sets. 
Theorem 2.19 (Jockusch and Stephan [7, Theorem 2.2(ii)]). Suppose that $\mathbf{a} \gg \mathbf{0}^{\prime}$. There exists an $r$-cohesive set $V$ such that $\operatorname{deg}(V)^{\prime} \leq \mathbf{a}$. Furthermore, every $r$ cohesive set $V$ satisfies $\operatorname{deg}(V)^{\prime} \gg \mathbf{0}^{\prime}$.

Using this result and a suitable r-cohesive set in place of the ultrafilter allows us to replace the 2-quantifier question of whether certain effectively given sets are infinite by a 1-quantifier question, and thus gives another proof that for every computable $B$ and computable $f:[B]^{n} \rightarrow p$, and any $\mathbf{a} \gg \mathbf{0}^{\prime}$, there exists a prehomogeneous pair $(A, g)$ for $f$ with $\operatorname{deg}(A \oplus g) \leq \mathbf{a}$. Hence, we get another proof of Theorem 2.10. However, by a much more detailed analysis of this approach for exponent 2, Cholak, Jockusch, and Slaman showed that it is also possible to force the jump of a homogeneous set in the construction.

Theorem 2.20 (Cholak, Jockusch, Slaman [2, essentially Lemma 4.6]). Suppose $p \geq 2, B \subseteq \omega$ is infinite, and $f:[B]^{2} \rightarrow p$. Suppose also that $B$ and $f$ are computable and that $\mathbf{a} \gg \mathbf{0}^{\prime}$. There exists a set $H$ homogeneous for $f$ such that $\operatorname{deg}(H)^{\prime} \leq \mathbf{a}$.

(Notice that in [2], Cholak, Jockusch, and Slaman essentially claim this theorem, but only prove the above result for stable $f$. However, one can make use of the fact that if $\mathbf{a} \gg \mathbf{0}^{\prime}$, then there exists a degree $\mathbf{b}$ such that $\mathbf{a} \gg \mathbf{b}$ and $\mathbf{b} \gg \mathbf{0}^{\prime}$ to get the desired conclusion from their arguments.) Furthermore, they showed that this characterization is sharp in the following sense.

Theorem 2.21 (Cholak, Jockusch, Slaman [2, Theorem 12.5]). There exists a computable $f:[\omega]^{2} \rightarrow 2$ such that $\operatorname{deg}(H)^{\prime} \gg \mathbf{0}^{\prime}$ for all sets $H$ homogeneous for $f$.

Therefore, as remarked on pp. 50-51 of [2], we obtain a corollary about Ramsey's Theorem for exponent 2 similar to Corollary 2.4 about König's Lemma with "jump universal" in place of "universal".

Corollary 2.22. There exists a computable $f:[\omega]^{2} \rightarrow 2$ such that that given any set $H_{f}$ homogeneous for $f$, and any computable $g:[\omega]^{2} \rightarrow 2$, there exists a set $H_{g}$ homogeneous for $g$ with $H_{g}^{\prime} \leq_{T} H_{f}^{\prime}$.

With the base case of exponent 2 settled, we can handle higher exponents. As the exponent increases, the bounds that we obtain in the Turing degrees increase by one jump each time.

We first show how we can use a relativization of Proposition 2.12 together with the Limit Lemma to lift results for exponent 2 to higher exponents. We state the theorem in relativized form to facilitate the inductive proof.

\section{Proposition 2.23.}

(1) Suppose that $n, p \geq 1, X \subseteq \omega, B \subseteq \omega$ is infinite, and $f:[B]^{n} \rightarrow p$. Suppose also that $B$ and $f$ are $X$-computable and that $\mathbf{a} \gg \operatorname{deg}(X)^{(n-1)}$. There exists a set $H$ homogeneous for $f$ such that $\operatorname{deg}(H)^{\prime} \leq \mathbf{a}$.

(2) For every $X \subseteq \omega$ and every $n \geq 2$, there exists an $X$-computable $f:[\omega]^{n} \rightarrow$ 3 such that for all sets $H$ homogeneous for $f$, we have $\operatorname{deg}(H \oplus X) \geq$ $\operatorname{deg}(X)^{(n-2)}$ and $\operatorname{deg}(H \oplus X)^{\prime} \gg \operatorname{deg}(X)^{(n-1)}$.

Proof. We prove the first statement by induction on $n$. The case $n=2$ follows by relativizing Theorem 2.20. Suppose that $n \geq 2$ and the result holds for $n$. Suppose that $B$ and $f:[B]^{n+1} \rightarrow p$ are $X$-computable, and $\mathbf{a} \gg \operatorname{deg}(X)^{(n)}$. Relativizing Proposition 2.9 to $X$, there exists a prehomogeneous pair $(A, g)$ for $f$ with 
$\operatorname{deg}(A \oplus g)^{\prime} \leq \operatorname{deg}(X)^{\prime \prime}$. By the inductive hypothesis, there exists a set $H$ homogeneous for $g:[A]^{n} \rightarrow p$ with $\operatorname{deg}(H)^{\prime} \leq \mathbf{a}$ since $\mathbf{a} \gg \operatorname{deg}(X)^{(n)}=\operatorname{deg}\left(X^{\prime \prime}\right)^{(n-2)} \geq$ $\left(\operatorname{deg}(A \oplus g)^{\prime}\right)^{(n-2)}=\operatorname{deg}(A \oplus g)^{(n-1)}$. By Claim 2.7, $H$ is homogeneous for $f$.

We prove the second part of the proposition in the following strong form. For every $X \subseteq \omega$ and every $n \geq 2$, there exists an $X$-computable $f:[\omega]^{n} \rightarrow 3$ such that for all sets $H$ homogeneous for $f$, we have $f\left([H]^{n}\right) \neq\{2\}, \operatorname{deg}(H \oplus X) \geq \operatorname{deg}(X)^{(n-2)}$ and $\operatorname{deg}(H \oplus X)^{\prime} \gg \operatorname{deg}(X)^{(n-1)}$. The case $n=2$ follows by relativizing Corollary 2.21. Suppose that $n \geq 2$ and the result holds for $n$. Fix an $X^{\prime}$-computable $g:[\omega]^{n} \rightarrow 3$ such that for all sets $H$ homogeneous for $g$, we have $g\left([H]^{n}\right) \neq\{2\}$, $\operatorname{deg}\left(H \oplus X^{\prime}\right) \geq \operatorname{deg}\left(X^{\prime}\right)^{(n-2)}=\operatorname{deg}(X)^{(n-1)}$ and $\operatorname{deg}\left(H \oplus X^{\prime}\right)^{\prime} \gg \operatorname{deg}\left(X^{\prime}\right)^{(n-1)}=$ $\operatorname{deg}(X)^{(n)}$. By the Limit Lemma, there is an $X$-computable $g_{1}:[\omega]^{n+1} \rightarrow 3$ such that $g(x)=\lim _{s} g_{1}(x, s)$ for all $x \in[\omega]^{n}$. Notice that if $H$ is homogeneous for $g_{1}$, then $H$ is homogeneous for $g$, and $g\left([H]^{n}\right)=g_{1}\left([H]^{n+1}\right)$. By Proposition 2.12 relativized to $X$ and the fact that $n+1 \geq 3$, there exists an $X$-computable $h:[\omega]^{n+1} \rightarrow 2$ such that for all infinite sets $H$ homogeneous for $h$, we have $h\left([H]^{n+1}\right)=\{0\}$ and $H \oplus X \geq_{T} X^{\prime}$. Define an $X$-computable $f:[\omega]^{n+1} \rightarrow 3$ by

$$
f(y)= \begin{cases}g_{1}(y) & \text { if } h(y)=0 \\ 2 & \text { if } h(y)=1\end{cases}
$$

Suppose that $H$ is homogeneous for $f$. If $f\left([H]^{n+1}\right)=\{2\}$, then for all $y \in[H]^{n+1}$, either $h(y)=1$ or $g_{1}(y)=2$. By Ramsey's Theorem applied to the function $h \uparrow[H]^{n+1}:[H]^{n+1} \rightarrow 2$, there exists an infinite set $I \subseteq H$ such that either $h\left([I]^{n+1}\right)=\{1\}$ or $h\left([I]^{n+1}\right)=\{0\}$, and hence $g\left([I]^{n}\right)=g_{1}\left([I]^{n+1}\right)=\{2\}$, both of which are impossible. Therefore, $f\left([H]^{n+1}\right) \neq\{2\}$, and hence $H$ is homogeneous for both $h$ and $g_{1}$. Since $H$ is homogeneous for $h$, we have $H \oplus X \geq_{T}$ $X^{\prime}$. Since every set homogeneous for $g_{1}$ is also homogeneous for $g$, we have $\operatorname{deg}\left(H \oplus X^{\prime}\right) \geq \operatorname{deg}(X)^{(n-1)}$ and $\operatorname{deg}\left(H \oplus X^{\prime}\right)^{\prime} \gg \operatorname{deg}(X)^{(n)}$. Hence, $f\left([H]^{n+1}\right) \neq$ $\{2\}, \operatorname{deg}(H \oplus X) \geq \operatorname{deg}\left(H \oplus X^{\prime}\right) \geq \operatorname{deg}(X)^{(n-1)}$ and $\operatorname{deg}(H \oplus X)^{\prime} \geq \operatorname{deg}\left(H \oplus X^{\prime}\right)^{\prime} \gg$ $\operatorname{deg}(X)^{(n)}$.

The following (unrelativized) question of whether we can replace the 3-coloring from the previous proposition by a 2 -coloring is open.

Question 2.24. For each $n \geq 3$, does there exist a computable $f:[\omega]^{n} \rightarrow 2$ such that for all sets $H$ homogeneous for $f$, we have $\operatorname{deg}(H) \geq \mathbf{0}^{(n-2)}$ and $\operatorname{deg}(H)^{\prime} \gg$ $\mathbf{0}^{(n-1)}$ ?

\section{A NEW PRoOf OF THE CANONICAL RAMSEY THEOREM}

One important lesson to glean from Section 2 is that we can often improve an effective analysis of a theorem by examining a genuinely different proof of the result. In the original inductive proof of the Canonical Ramsey Theorem (see [3]), in order to prove the result for exponent $n \geq 2$, Erdös and Rado used Ramsey's Theorem for exponent $2 n$ together with the Canonical Ramsey Theorem for exponent $n-1$. Using Theorem 2.11, an effective analysis of their proof gives the result that if $B$ is computable and $f:[B]^{2} \rightarrow \omega$ is computable, then there exists a $\Pi_{4}^{0}$ set canonical for $f$. However, as $n$ increases, the use of induction causes the arithmetical bounds to grow on the order of $n^{2}$. Rado [15] discovered a noninductive proof of the Canonical Ramsey Theorem which still used Ramsey's Theorem for exponent $2 n$ to prove the result for exponent $n$. An effective analysis of his proof shows that given $n \geq 2$, a 
computable $B$, and a computable $f:[B]^{n} \rightarrow \omega$, there exists a $\Delta_{2 n+1}^{0}$ set canonical for $f$.

We give a new proof of the Canonical Ramsey Theorem which is inductive and similar in broad outline to the proofs of Ramsey's Theorem sketched above. The basic question is how to define a "precanonical pair" $(A, g)$ so that we can carry out the same outline to prove the Canonical Ramsey Theorem. For simplicity, consider a function $f:[\omega]^{2} \rightarrow \omega$. We will enumerate $A$ in increasing order as $a_{0}, a_{1}, \ldots$ We begin by letting $a_{0}=0$. If there exists $c \in \omega$ such that there are infinitely many $b \in \omega$ with $f\left(a_{0}, b\right)=c$, then we can define $g\left(a_{0}\right)=c$, restrict attention to the set $I_{0}=\left\{b \in \omega: f\left(a_{0}, b\right)=c\right\}$, and after letting $a_{1}=\min I_{0}$, continue in this fashion. In this case, we've made progress toward achieving a $u$-canonical set with $1 \notin u$, because if we fix $a_{0}$ and vary $b \in I_{0}$, we do not change the value of $f$. If we succeed infinitely many times in this manner with a fixed $c$, then the corresponding elements form a $\emptyset$-canonical set, while if we succeed with infinitely many different $c$ in this manner, then the corresponding elements form a $\{0\}$-canonical set. Notice that this decision (one fixed $c$ versus infinitely many distinct $c$ ) amounts to finding a canonical set for exponent 1 for $g$ restricted to the set of successes.

The problem arises when for each $c \in \omega$, there are only finitely many $b \in \omega$ with $f\left(a_{0}, b\right)=c$. Now we must seek to make progress toward achieving a $u$-canonical set with $1 \in u$. We therefore let $I_{0}=\left\{b \in \omega: f\left(a_{0}, b\right) \neq f\left(a_{0}, b^{\prime}\right)\right.$ for all $\left.b^{\prime}<b\right\}$, so that if we fix $a_{0}$ and vary $b \in I_{0}$, we always change the value of $f$. We now want to let $g\left(a_{0}\right)$ be some new, infinitary color $d$ distinct from each $c \in \omega$. Suppose that we then set $a_{1}=\min I_{0}$, and again are faced with the situation that for each $c \in \omega$, there are only finitely many $b \in I_{0}$ with $f\left(a_{1}, b\right)=c$. We first want to thin out $I_{0}$ to an infinite set $I_{0}^{\prime}$ so that $f\left(a_{i}, b_{0}\right)=f\left(a_{j}, b_{1}\right) \rightarrow b_{0}=b_{1}$ whenever $0 \leq i, j \leq 1$ and $b_{0}, b_{1} \in I_{0}^{\prime}$ (which is possible by the assumption on $a_{0}, a_{1}$ ). This allows both $a_{0}$ and $a_{1}$ to be in the same $u$-canonical set with $1 \in u$. Next, we need to assign an appropriate infinitary color to $g\left(a_{1}\right)$ so that a canonical set for $g$ will be a $u$-canonical set for $f$. Thus, if the set $\left\{b \in I_{0}^{\prime}: f\left(a_{0}, b\right)=f\left(a_{1}, b\right)\right\}$ is infinite, we let $g\left(a_{1}\right)=g\left(a_{0}\right)$ and we let $I_{1}$ be this set. Otherwise we will set $g\left(a_{1}\right)$ to a new infinitary color and let $I_{1}=\left\{b \in I_{0}^{\prime}: f\left(a_{0}, b\right) \neq f\left(a_{1}, b\right)\right\}$. If we succeed infinitely many times in this manner with a fixed infinitary color $d$, then the corresponding elements form a $\{1\}$-canonical set, while if we succeed with infinitely many different $d$ in this manner, then the corresponding elements form a $\{0,1\}$-canonical set. Notice again that this decision (one fixed $d$ versus infinitely many distinct $d$ ) amounts to finding a canonical set for exponent 1 for $g$ restricted to those elements assigned infinitary colors.

In general, given $f:[B]^{n+1} \rightarrow \omega$, we can pursue the above strategy to get an infinite set $A \subseteq B$ and a function $g:[A]^{n} \rightarrow \omega \times 2$, where we interpret each $(c, 0) \in \omega \times 2$ as a finitary color and each $(d, 1) \in \omega \times 2$ as an infinitary color. Now, before we can apply induction, it is important to thin out our set $A$ to a set $D$ so that either $g$ maps all elements of $[D]^{n}$ to finitary colors, or $g$ maps all elements of $[D]^{n}$ to infinitary colors. Of course, we can do this with a simple application of Ramsey's Theorem for exponent $n$. Although this strategy will succeed in proving the Canonical Ramsey Theorem, the use of Ramsey's Theorem is costly to an effective analysis. We therefore pursue a slightly different approach which will roll this use of Ramsey's Theorem into the induction. Hence, we extend the notion of canonical sets to functions $f:[B]^{n} \rightarrow \omega \times p$ for $p \in \omega$ by also stipulating that a 
canonical $C$ set must have the property that $f$ maps all elements of $[C]^{n}$ into the same column of $\omega \times p$. With this modification in place, the above strategy will give us an infinite set $A$ and a function $g:[A]^{n} \rightarrow \omega \times 2 p$, where we interpret each $(c, q) \in \omega \times 2 p$ with $0 \leq q<p$ as a finitary color corresponding to column $q$ and each $(d, q) \in \omega \times 2 p$ with $p \leq q<2 p$ as an infinitary color corresponding to column $q-p$ of $\omega \times p$. Applying induction to this $g$ will give us the result because the resulting canonical set will be mapped by $g$ entirely into one column of $\omega \times 2 p$.

Definition 3.1. Let $\pi_{1}: \omega \times \omega \rightarrow \omega$ be projection onto the first coordinate and let $\pi_{2}: \omega \times \omega \rightarrow \omega$ be projection onto the second coordinate.

Definition 3.2. Suppose that $n, p \geq 1, B \subseteq \omega$ is infinite, $f:[B]^{n} \rightarrow \omega \times p$, and $u \subseteq n$. We say that a set $C$ is $u$-canonical for $f$ if:

(1) $C \subseteq B$.

(2) $C$ is infinite.

(3) $C$ is homogeneous for $\pi_{2} \circ f:[B]^{n} \rightarrow p$.

(4) If $x_{1}, x_{2} \in[C]^{n}$, then $f\left(x_{1}\right)=f\left(x_{2}\right) \leftrightarrow x_{1}\left\lceil u=x_{2}\lceil u\right.$.

We say that a set $C$ is canonical for $f$ if there exists $u \subseteq n$ such that $C$ is $u$-canonical for $f$.

Remark 3.3. Suppose that $n \geq 1, B \subseteq \omega$ is infinite, $f:[B]^{n} \rightarrow \omega$, and $u \subseteq n$. Define $f^{*}:[B]^{n} \rightarrow \omega \times 1$ by letting $f^{*}(x)=(f(x), 0)$. Notice that for any infinite set $C \subseteq \omega, C$ is $u$-canonical for $f$ (as in Definition 1.6) if and only if $C$ is $u$ canonical for $f^{*}$ (as in Definition 3.2). Therefore, in the following, we identify a function $f:[B]^{n} \rightarrow \omega$ with the corresponding function $f^{*}:[B]^{n} \rightarrow \omega \times 1$.

For the reasons mentioned above, we prove the Canonical Ramsey Theorem by induction on $n$ in the following strong form.

Theorem 3.4. Suppose that $n, p \geq 1, B \subseteq \omega$ is infinite, and $f:[B]^{n} \rightarrow \omega \times p$. There exists a set $C \subseteq B$ such that $C$ is canonical for $f$.

Definition 3.5. Suppose that $n, p \geq 1, B \subseteq \omega$ is infinite, and $f:[B]^{n+1} \rightarrow \omega \times p$. We call a pair $(A, g)$, where $A \subseteq B$ is infinite and $g:[A]^{n} \rightarrow \omega \times 2 p$, a precanonical pair for $f$ if:

(1) For all $x \in[A]^{n}$ with $g(x)=(c, q)$ where $0 \leq q<p$, we have $f(x, a)=(c, q)$ for all $a \in A$ with $a>x$.

(2) For all $x \in[A]^{n}$ with $g(x)=(d, q)$ where $p \leq q<2 p$, we have $\pi_{2}(f(x, a))=$ $q-p$ for all $a \in A$ with $a>x$.

(3) For all $x_{1}, x_{2} \in[A]^{n}$ with $g\left(x_{1}\right)=\left(d_{1}, q\right)$ and $g\left(x_{2}\right)=\left(d_{2}, q\right)$ where $p \leq q<$ $2 p$, and all $a_{1}, a_{2} \in A$ with $a_{1}>x_{1}$ and $a_{2}>x_{2}$,

(a) If $a_{1} \neq a_{2}$, then $f\left(x_{1}, a_{1}\right) \neq f\left(x_{2}, a_{2}\right)$.

(b) If $a_{1}=a_{2}$, then $f\left(x_{1}, a_{1}\right)=f\left(x_{2}, a_{2}\right) \leftrightarrow d_{1}=d_{2}$.

We first show that the above definition of "precanonical pair" allows our outline to succeed.

Claim 3.6. Suppose that $n, p \geq 1, B \subseteq \omega$ is infinite, $f:[B]^{n+1} \rightarrow \omega \times p$, and $(A, g)$ is a precanonical pair for $f$. Suppose that $C \subseteq A$ is $u$-canonical for $g$, where $u \subseteq n$.

(1) If $\left.\left(\pi_{2} \circ g\right)\left([C]^{n}\right)\right)=\{q\}$ where $0 \leq q<p$, then $C$ is $u$-canonical for $f$ (now viewing $u$ as a subset of $n+1)$. 
(2) If $\left.\left(\pi_{2} \circ g\right)\left([C]^{n}\right)\right)=\{q\}$ where $p \leq q<2 p$, then $C$ is $(u \cup\{n\})$-canonical for $f$.

Proof. (1) For any $x \in[C]^{n}$ and $a \in C$ with $x<a$, we have $\pi_{2}(f(x, a))=$ $\pi_{2}(g(x))=q$ by condition (1) of Definition 3.5; hence $C$ is homogeneous for $\pi_{2} \circ f$. Let $x_{1}, x_{2} \in[C]^{n}, a_{1}, a_{2} \in C$ with $x_{1}<a_{1}$ and $x_{2}<a_{2}$. By condition (1) of Definition 3.5, we have $f\left(x_{1}, a_{1}\right)=g\left(x_{1}\right)$ and $f\left(x_{2}, a_{2}\right)=g\left(x_{2}\right)$. Therefore, $f\left(x_{1}, a_{1}\right)=f\left(x_{2}, a_{2}\right) \leftrightarrow g\left(x_{1}\right)=g\left(x_{2}\right) \leftrightarrow x_{1} \uparrow u=x_{2} \uparrow u$. Hence, $C$ is $u$-canonical for $f$.

(2) For any $x \in[C]^{n}$ and $a \in C$ with $x<a$, we have $\pi_{2}(f(x, a))=q-p$ by condition (2) of Definition 3.5; hence $C$ is homogeneous for $\pi_{2} \circ f$. Let $x_{1}, x_{2} \in[C]^{n}$, $a_{1}, a_{2} \in C$ with $x_{1}<a_{1}$ and $x_{2}<a_{2}$. Suppose first that $x_{1} \uparrow u=x_{2} \uparrow u$ and $a_{1}=a_{2}$. Then $g\left(x_{1}\right)=g\left(x_{2}\right)$ (since $C$ is $u$-canonical for $g$ ) and $a_{1}=a_{2}$. Therefore, by condition (3b) of Definition 3.5, we have $f\left(x_{1}, a_{1}\right)=f\left(x_{2}, a_{2}\right)$. Suppose now that either $x_{1}\left\lceil u \neq x_{2} \uparrow u\right.$ or $a_{1} \neq a_{2}$. If $a_{1} \neq a_{2}$, then $f\left(x_{1}, a_{1}\right) \neq f\left(x_{2}, a_{2}\right)$ by condition (3a) of Definition 3.5. If $x_{1}\left\lceil u \neq x_{2}\left\lceil u\right.\right.$ and $a_{1}=a_{2}$, then $g\left(x_{1}\right) \neq g\left(x_{2}\right)$ (since $C$ is $u$-canonical for $g$ ) and $a_{1}=a_{2}$, so $f\left(x_{1}, a_{1}\right) \neq f\left(x_{2}, a_{2}\right)$ by condition (3b) of Definition 3.5. Therefore, $f\left(x_{1}, a_{1}\right)=f\left(x_{2}, a_{2}\right)$ if and only if $x_{1} \uparrow u=x_{2} \uparrow u$ and $a_{1}=a_{2}$, so $C$ is $(u \cup\{n\})$-canonical for $f$.

Next, we show that precanonical pairs exist by a method along the lines of the standard proof of Ramsey's Theorem. We build a precanonical pair $(A, g)$ in stages which consist of selecting a new element for $A$ and thinning out the set of potential later elements to make them acceptable to the new element and its chosen color. To facilitate this construction, we first define a notion of precanonical triple which will provide an approximation to a desired precanonical pair.

Definition 3.7. If $a \in \omega$ and $Z \subseteq \omega$ with $Z \neq \emptyset$, we write $a<Z$ to mean that $a<b$ for every $b \in Z$.

Definition 3.8. Suppose that $n, p \geq 1, B \subseteq \omega$ is infinite, and $f:[B]^{n+1} \rightarrow \omega \times p$. We call a triple $(z, I, g)$ where $z \subseteq B$ is finite, $I \subseteq B$ is infinite, $z<I$, and $g:[z]^{n} \rightarrow \omega \times 2 p$, a precanonical triple for $f$ if:

(1) For all $x \in[z]^{n}$ with $g(x)=(c, q)$ where $0 \leq q<p$, we have $f(x, a)=(c, q)$ for all $a \in z \cup I$ with $a>x$.

(2) For all $x \in[z]^{n}$ with $g(x)=(d, q)$ where $p \leq q<2 p$, we have $\pi_{2}(f(x, a))=$ $q-p$ for all $a \in z \cup I$ with $a>x$.

(3) For all $x_{1}, x_{2} \in[z]^{n}$ with $g\left(x_{1}\right)=\left(d_{1}, q\right)$ and $g\left(x_{2}\right)=\left(d_{2}, q\right)$ where $p \leq q<$ $2 p$, and all $a_{1}, a_{2} \in z \cup I$ with $a_{1}>x_{1}$ and $a_{2}>x_{2}$,

(a) If $a_{1} \neq a_{2}$, then $f\left(x_{1}, a_{1}\right) \neq f\left(x_{2}, a_{2}\right)$.

(b) If $a_{1}=a_{2}$, then $f\left(x_{1}, a_{1}\right)=f\left(x_{2}, a_{2}\right) \leftrightarrow d_{1}=d_{2}$.

We will make use of the following ordering in the construction to allow us to easily refer to work carried out in previous (sub)stages.

Definition 3.9. For each $n \in \omega$, we define a total ordering $<_{n}$ of $[\omega]^{n}$ as follows. For $x, y \in[\omega]^{n}$, we let $x<_{n} y$ if and only if $x \neq y$ and $x(i)<y(i)$, where $i$ is the greatest integer less than $n$ with $x(i) \neq y(i)$.

Proposition 3.10. Suppose that $n, p \geq 1, B \subseteq \omega$ is infinite, and $f:[B]^{n+1} \rightarrow$ $\omega \times p$. There exists a precanonical pair $(A, g)$ for $f$. 
Proof. We inductively define a sequence $\left(a_{m}, I_{m}, g_{m}\right)_{m \in \omega}$ such that:

- $a_{m} \in B$.

- $I_{m} \subseteq B$ is infinite.

- $g_{m}:\left[\left\{a_{i}: i \leq m\right\}\right]^{n} \rightarrow \omega \times 2 p$.

- $a_{0}<a_{1}<\cdots<a_{m-1}<a_{m}<I_{m} \subseteq I_{m-1} \subseteq \cdots \subseteq I_{1} \subseteq I_{0}$.

- $g_{0} \subseteq g_{1} \subseteq \cdots \subseteq g_{m}$.

- $\left(\left\{a_{i}: i \leq m\right\}, I_{m}, g_{m}\right)$ is a precanonical triple for $f$.

We begin by letting $a_{-1}=-1, I_{-1}=B$, and $g_{-1}=\emptyset$. Suppose that we have defined our sequence through stage $m \geq-1$. We first let $a_{m+1}=\min \left(I_{m}\right)$, $I_{m}^{\prime}=I_{m}-\left\{a_{m+1}\right\}$, and $g_{m+1}(x)=g_{m}(x)$ for all $x \in\left[\left\{a_{i}: i \leq m\right\}\right]^{n}$. Let $\mathcal{F}=$ $\left[\left\{a_{i}: i \leq m+1\right\}\right]^{n}$, and list the elements of $\mathcal{F}$ whose greatest element is $a_{m+1}$ as $x_{0}<_{n} x_{1}<_{n} \cdots<_{n} x_{\ell-1}$, where $\ell=\left(\begin{array}{c}m+1 \\ n-1\end{array}\right)$. If $\ell=0$, let $I_{m+1}=I_{m}^{\prime}$ and $g_{m+1}=g_{m}$. Otherwise, we proceed inductively through the $x_{k}$, defining $g_{m+1}\left(x_{k}\right)$ and infinite sets $J_{0}, J_{1}, \ldots, J_{\ell}$ such that $I_{m}^{\prime}=J_{0} \supseteq J_{1} \supseteq \cdots \supseteq J_{\ell}$ along the way. Let $J_{0}=I_{m}^{\prime}$. Suppose that $k<\ell$ and we have defined $J_{k}$. First, since $J_{k}$ is infinite, there exists $q<p$ such that there are infinitely many $b \in J_{k}$ with $\pi_{2}\left(f\left(x_{k}, b\right)\right)=q$. Fix the least such $q$, and let $H_{k}=\left\{b \in J_{k}: \pi_{2}\left(f\left(x_{k}, b\right)\right)=q\right\}$.

Case 1: There exists $c \in \omega$ such that there are infinitely many $b \in H_{k}$ with $f\left(x_{k}, b\right)=(c, q)$. In this case, let $g_{m+1}\left(x_{k}\right)=(c, q)$ and let $J_{k+1}=\left\{b \in H_{k}\right.$ : $\left.f\left(x_{k}, b\right)=(c, q)\right\}$. Proceed to the next value of $k<\ell$, if it exists.

Case 2: Otherwise, for every $c \in \omega$, there are only finitely many $b \in H_{k}$ with $f\left(x_{k}, b\right)=(c, q)$. Let $\mathcal{D}=\left\{y \in \mathcal{F}: y<_{n} x_{k}\right.$ and $\left.\pi_{2}\left(g_{m+1}(y)\right)=q+p\right\}$, and notice that for each $y \in \mathcal{D}$ and each $c \in \omega$, there is at most one $b \in H_{k}$ with $f(y, b)=(c, q)$ (if $\max (y)<a_{m+1}$, this follows from the fact that $\left(\left\{a_{i}: i \leq m\right\}, I_{m}, g_{m}\right)$ is a precanonical triple for $f$ and $H_{k} \subseteq I_{m}$, while if $\max (y)=a_{m+1}$, say $y=x_{i}$ with $1 \leq i<k$, this follows from the fact that $H_{k} \subseteq J_{i+1}$, so $f\left(y, b_{1}\right) \neq f\left(y, b_{2}\right)$ for all $b_{1}, b_{2} \in H_{k}$ with $b_{1} \neq b_{2}$ by construction). We now inductively define an increasing $h: \omega \rightarrow H_{k}$ such that $f\left(x_{k}, h(i)\right) \neq f(y, h(j))$ whenever $i \neq j \in \omega$ and $y \in \mathcal{D} \cup\left\{x_{k}\right\}$. Let $h(0)=\min \left(H_{k}\right)$. Suppose that we have defined $h(t)$. By the assumption of Case 2 and the above comments, there exists $b \in H_{k}$ with $b>h(t)$ such that $f\left(x_{k}, b\right) \notin\left\{f(y, h(i)): y \in \mathcal{D} \cup\left\{x_{k}\right\}, 0 \leq i \leq t\right\}$ and $f(y, b) \notin\left\{f\left(x_{k}, h(i)\right): 0 \leq i \leq\right.$ $t\}$ for all $y \in \mathcal{D}$ (since each of these sets is finite), and we let $h(t+1)$ be the least such $b$. Let $H_{k}^{\prime}=\{h(t): t \in \omega\}$.

Subcase 1: There exists $y \in \mathcal{D}$ such that $\left\{b \in H_{k}^{\prime}: f\left(x_{k}, b\right)=f(y, b)\right\}$ is infinite. In this case, choose the least such $y$ (under the ordering $<_{n}$ ), let $g_{m+1}\left(x_{k}\right)=$ $g_{m+1}(y)$, and let $J_{k+1}=\left\{b \in H_{k}^{\prime}: f\left(x_{k}, b\right)=f(y, b)\right\}$. Proceed to the next value of $k<\ell$, if it exists.

Subcase 2: Otherwise, for every $y \in \mathcal{D}$, there are only finitely many $b \in H_{k}^{\prime}$ with $f\left(x_{k}, b\right)=f(y, b)$. Thus, there are only finitely many $b \in H_{k}^{\prime}$ such that there exists $y \in \mathcal{D}$ with $f\left(x_{k}, b\right)=f(y, b)$. Let $g_{m+1}\left(x_{k}\right)=(d, q+p)$, where $d$ is least such that $g_{m+1}(y) \neq(d, q+p)$ for all $y \in \mathcal{D}$ and let $J_{k+1}=\left\{b \in H_{k}^{\prime}: f\left(x_{k}, b\right) \neq f(y, b)\right.$ for all $y \in \mathcal{D}\}$. Proceed to the next value of $k<\ell$, if it exists.

Once we've run through all $k<\ell$ and defined $J_{\ell}$, set $I_{m+1}=J_{\ell}$. One easily checks that the invariants are maintained (i.e. that $a_{m}<a_{m+1}<I_{m+1} \subseteq I_{m}$, $g_{m} \subseteq g_{m+1}$, and $\left(\left\{a_{i}: i \leq m+1\right\}, I_{m+1}, g_{m+1}\right)$ is a precanonical triple for $\left.f\right)$. This completes stage $m+1$.

Finally, let $A=\left\{a_{m}: m \in \omega\right\}$ and $g=\bigcup_{m \in \omega} g_{m}$. Then $(A, g)$ is a precanonical pair for $f$. 
Proof of Theorem 3.4. The proof is by induction on $n$. Suppose that $n=1$ so that we have $f:[B]^{1} \rightarrow \omega \times p$. Fix an infinite $A \subseteq B$ and $q<p$ such that $\pi_{2}\left(f\left([A]^{1}\right)\right)=\{q\}$. If there exists a $c \in \omega$ such that there are infinitely many $a \in A$ with $f(a)=(c, q)$, let $C=\{a \in A: f(a)=(c, q)\}$, and notice that $f\left(a_{1}\right)=f\left(a_{2}\right)$ for all $a_{1}, a_{2} \in C$, so $C$ is $\emptyset$-canonical for $f$. Otherwise, there are infinitely many $c \in \omega$ such that there is an $a \in A$ with $f(a)=(c, q)$. Letting $C=\{a \in A: f(a) \neq f(b)$ for all $b<a$ with $b \in A\}$, we see that $f\left(a_{1}\right) \neq f\left(a_{2}\right)$ for all $a_{1}, a_{2} \in C$, so $C$ is $\{0\}$-canonical for $f$.

Suppose that the theorem holds for $n$, and we're given $f:[B]^{n+1} \rightarrow \omega \times p$. By Proposition 3.10, there exists a precanonical pair $(A, g)$ for $f$. Applying the inductive hypothesis to $g:[A]^{n} \rightarrow \omega \times 2 p$, there exists $C \subseteq A$ which is canonical for $g$. By Claim 3.6, $C$ is canonical for $f$.

\section{Computability-theoretic analysis}

If we analyze the proof of Proposition 3.10 for a given computable $B$ and computable $f:[B]^{n+1} \rightarrow \omega \times p$, we can easily see that there exists a precanonical pair $(A, g)$ for $f$ with $A \oplus g \leq_{T} 0^{\prime \prime \prime}$. It seems that we need a $0^{\prime \prime \prime}$-oracle to decide the 3 quantifier $(\exists \forall \exists)$ question of whether to enter Case 1 or Case 2. However, by making use of an r-cohesive set, we can lower the complexity to a 2-quantifier question.

Recall the characterization of the Turing degrees of jumps of r-cohesive sets from Theorem 2.19. The Low Basis Theorem relative to $\mathbf{0}^{\prime}$ yields an $\mathbf{a} \gg \mathbf{0}^{\prime}$ such that $\mathbf{a}^{\prime}=\mathbf{0}^{\prime \prime}$. Using this $\mathbf{a}$ in Theorem 2.19 gives the following corollary.

Corollary 4.1 (Jockusch and Stephan [7]). There exists an $r$-cohesive set $V$ such that $V^{\prime \prime} \leq_{T} 0^{\prime \prime}$.

Below, we will need r-cohesive sets of low complexity inside a given infinite computable set. The following easy lemma provides these.

Definition 4.2. Given an infinite set $B$, we let $p_{B}: \omega \rightarrow \omega$ be the function enumerating $B$ in increasing order (so $p_{B}(n)$ is the $(n+1)^{\text {st }}$ element of $B$ ).

Lemma 4.3. Suppose that $B$ is an infinite computable set. If $V$ is $r$-cohesive, then $p_{B}(V) \subseteq B$ is r-cohesive and $p_{B}(V) \equiv_{T} V$.

Proof. Notice that $p_{B}(V) \subseteq B$ is infinite and $p_{B}(V) \equiv_{T} V$ because $p_{B}$ is computable and strictly increasing. Let $Z$ be a computable set. Since $V$ is r-cohesive and $p_{B}^{-1}(Z)$ is computable, either $V \cap p_{B}^{-1}(Z)$ is finite or $V \cap \overline{p_{B}^{-1}(Z)}$ is finite. If $V \cap p_{B}^{-1}(Z)$ is finite, then $V \subseteq \subseteq^{*} \overline{p_{B}^{-1}(Z)}$, so $p_{B}(V) \subseteq^{*} p_{B}\left(\overline{p_{B}^{-1}(Z)}\right) \subseteq \bar{Z}$, and hence $p_{B}(V) \cap Z$ is finite. If $V \cap \overline{p_{B}^{-1}(Z)}$ is finite, then $V \subseteq^{*} p_{B}^{-1}(Z)$, so $p_{B}(V) \subseteq^{*}$ $p_{B}\left(p_{B}^{-1}(Z)\right) \subseteq Z$, and hence $p_{B}(V) \cap \bar{Z}$ is finite. It follows that $p_{B}(V)$ is rcohesive.

Proposition 4.4. Suppose that $n, p \geq 1, B \subseteq \omega$ is infinite, and $f:[B]^{n+1} \rightarrow \omega \times p$. Suppose also that $B$ and $f$ are computable. There exists a precanonical pair $(A, g)$ for $f$ such that $A \oplus g \leq_{T} 0^{\prime \prime}$. Furthermore, if $n=1$ and $\mathbf{a} \gg \mathbf{0}^{\prime}$, there exists a precanonical pair $(A, g)$ for $f$ with $\operatorname{deg}(A \oplus g) \leq \mathbf{a}$.

Proof. By Corollary 4.1 and Lemma 4.3, there exists an r-cohesive set $V \subseteq B$ such that $V^{\prime \prime} \leq_{T} 0^{\prime \prime}$. For each $x \in[V]^{n}$ and each $(c, q) \in \omega \times p$, the set $Z_{x,(c, q)}=\{b \in$ $B: f(x, b)=(c, q)\}$ is computable, so either $V \cap Z_{x,(c, q)}$ is finite or $V \cap \overline{Z_{x,(c, q)}}$ is finite. 
We now carry out the above existence proof of a precanonical pair for $f \uparrow$ $[V]^{n+1}:[V]^{n+1} \rightarrow \omega \times p$ using a $V^{\prime \prime}$-oracle and characteristic indices (relative to $V)$ for all infinite sets. As we proceed through the proof, the first noncomputable (relative to $V$ ) step is the construction of $H_{k}$, where we need to find the least $q<p$ such that $H_{k}=\left\{b \in J_{k}: \pi_{2}\left(f\left(x_{k}, b\right)\right)=q\right\}$ is infinite, which we can do using a $V^{\prime \prime}$-oracle. Next, we need to decide whether to enter Case 1 or Case 2. By the last sentence of the above paragraph, we enter Case 1 if and only if $(\exists c)(\exists m)(\forall b)\left[\left(b \in H_{k} \wedge b \geq m\right) \rightarrow f\left(x_{k}, b\right)=(c, q)\right]$. Again, we can decide this question using a $V^{\prime \prime}$-oracle. If we enter Case 2, the next noncomputable (relative to $V$ ) step is the decision whether to enter Subcase 1 or Subcase 2. Since $\mathcal{D}$ is finite, and for each $y \in \mathcal{D}$ we need to determine whether a given $V$-computable set is infinite, we can again decide this question using a $V^{\prime \prime}$-oracle. The rest of the steps of the proof are $V$-computable, so we end up with a precanonical pair $(A, g)$ for $f\left\lceil[V]^{n+1}\right.$ (hence for $f$ ) such that $A \oplus g \leq_{T} V^{\prime \prime} \leq_{T} 0^{\prime \prime}$.

Suppose now that $n=1$ and $\mathbf{a} \gg \mathbf{0}^{\prime}$. By Theorem 2.19 and Lemma 4.3, there exists an r-cohesive set $V \subseteq B$ such that $\operatorname{deg}(V)^{\prime} \leq \mathbf{a}$. For each $a \in V$ and $q<p$, the set $Z_{a, q}=\left\{b \in B: \pi_{2}(f(a, b))=q\right\}$ is computable, so either $V \cap Z_{a, q}$ is finite or $V \cap \overline{Z_{a, q}}$ is finite. Therefore, for each $a \in V, \lim _{b \in V} \pi_{2}(f(a, b))$ exists, and we denote its value by $q_{a}$. Notice that we can use a $V^{\prime}$-oracle to compute $q_{a}$ given $a \in V$. Similarly, for each $a \in V$ and $c \in \omega$, the set $Z_{a, c}=\left\{b \in B: \pi_{1}(f(a, b))=c\right\}$ is computable, so either $V \cap Z_{a, c}$ is finite or $V \cap \overline{Z_{a, c}}$ is finite. Therefore, for each $a \in V$, either $\lim _{b \in V} \pi_{1}(f(a, b))$ exists (and is finite) or $\lim _{b \in V} \pi_{1}(f(a, b))=\infty$.

Let $Y=\left\{a \in V: \lim _{b \in V} \pi_{1}(f(a, b))<\infty\right\}$. Notice that $a \in Y$ if and only if $(\exists c)(\exists m)(\forall b)\left[(b \geq m \wedge b \in V) \rightarrow \pi_{1}(f(a, b))=c\right]$; hence $Y \in \Sigma_{2}^{0, V}$.

Case 1: $Y$ is infinite: Fix an infinite $I \subseteq Y$ such that $I \leq_{T} V^{\prime}$. For each $a \in V$, we can use a $V^{\prime}$-oracle to determine whether $a \in I$, and if so to compute $c_{a}=\lim _{b \in V} \pi_{1}(f(a, b))$. We now construct a precanonical pair $(A, g)$ for $f$ using a $V^{\prime}$-oracle. First, let $a_{0}$ be the least element of $I$ and let $g\left(a_{0}\right)=\left(c_{a_{0}}, q_{a_{0}}\right)$. If we have already defined $a_{0}, a_{1}, \ldots, a_{m}$, let $a_{m+1}$ be the least $b \in I$ such that $b>a_{m}$ and $f\left(a_{i}, b\right)=g\left(a_{i}\right)=\left(c_{a_{i}}, q_{a_{i}}\right)$ for all $i$ with $0 \leq i \leq m$, and let $g\left(a_{m+1}\right)=$ $\left(c_{a_{m+1}}, q_{a_{m+1}}\right)$. Letting $A=\left\{a_{m}: m \in \omega\right\}$, we see that $(A, g)$ is a precanonical pair for $f \uparrow[V]^{2}$ (hence for $f$ ) such that $\operatorname{deg}(A \oplus g) \leq \operatorname{deg}(V)^{\prime} \leq \mathbf{a}$.

Case 2: $Y$ is finite: Fix $\alpha$ such that $\lim _{b \in V} \pi_{1}(f(a, b))=\infty$ for all $a \in V$ with $a>\alpha$. We now construct a precanonical pair $(A, g)$ for $f$ using a $V^{\prime}$-oracle. First, let $a_{0}$ be the least element of $V$ greater than $\alpha$, and let $g\left(a_{0}\right)=\left(0, p+q_{a_{0}}\right)$. Suppose that we have already defined $a_{0}, a_{1}, \ldots, a_{m}$ and $g\left(a_{0}\right), g\left(a_{1}\right), \ldots, g\left(a_{m}\right)$, and assume inductively that for all sufficiently large $b \in V$, we have:

(1) For all $i \leq m, \pi_{2}\left(f\left(a_{i}, b\right)\right)=q_{a_{i}}$.

(2) For all $i, j, k \leq m$ with $i<k$ and $q_{a_{i}}=q_{a_{j}}, f\left(a_{i}, a_{k}\right) \neq f\left(a_{j}, b\right)$.

(3) For all $i, j \leq m$ with $q_{a_{i}}=q_{a_{j}}, f\left(a_{i}, b\right)=f\left(a_{j}, b\right) \leftrightarrow g\left(a_{i}\right)=g\left(a_{j}\right)$.

Using a $V^{\prime}$-oracle, let $a_{m+1}$ be the least $b \in V$ such that $b>a_{m}$ and (1), (2), and (3) hold for $b$. Let $D=\left\{i \in \omega: 0 \leq i \leq m\right.$ and $\left.q_{a_{i}}=q_{a_{m+1}}\right\}$. Notice that we can find (a canonical index for) $D$ using a $V^{\prime}$-oracle. For each $i \in D$, the set $Z_{i}=\left\{b \in B: b>a_{m+1}\right.$ and $\left.f\left(a_{i}, b\right)=f\left(a_{m+1}, b\right)\right\}$ is computable, so either $V \cap Z_{i}$ is finite or $V \cap \overline{Z_{i}}$ is finite. Also, the set $Z_{\infty}=\left\{b \in B: b>a_{m+1}\right.$ and $\left.f\left(a_{m+1}, b\right) \notin\left\{f\left(a_{i}, b\right): i \in D\right\}\right\}$ is computable, so either $V \cap Z_{\infty}$ is finite or $V \cap \overline{Z_{\infty}}$ is finite. Putting this together with the fact that the sets in the list $\left(V \cap Z_{i}\right)_{i \in D \cup\{\infty\}}$ are pairwise disjoint and have union equal to $\left\{b \in V: b>a_{m+1}\right\}$, it follows that 
there exists exactly one $j \in D \cup\{\infty\}$ with $V \cap \overline{Z_{j}}$ finite. Moreover, we can find this $j$ using a $V^{\prime}$-oracle (by running through $\beta \in B$ in increasing order and asking a $V^{\prime}$-oracle if all elements of $V$ greater than $\beta$ lie in one fixed $\left.Z_{i}\right)$. If $j \in D$, let $g\left(a_{m+1}\right)=g\left(a_{j}\right)$, and if $j=\infty$, let $g\left(a_{m+1}\right)=\left(d, p+q_{a_{m+1}}\right)$, where $d$ is the least element of $\omega-\left\{\pi_{1}\left(g\left(a_{i}\right)\right): i \in D\right\}$. Then for all sufficiently large $b \in V$, we have:

(1) For all $i \leq m+1, \pi_{2}\left(f\left(a_{i}, b\right)\right)=q_{a_{i}}$.

(2) For all $i, j, k \leq m+1$ with $i<k$ and $q_{a_{i}}=q_{a_{j}}, f\left(a_{i}, a_{k}\right) \neq f\left(a_{j}, b\right)$.

(3) For all $i, j \leq m+1$ with $q_{a_{i}}=q_{a_{j}}, f\left(a_{i}, b\right)=f\left(a_{j}, b\right) \leftrightarrow g\left(a_{i}\right)=g\left(a_{j}\right)$.

Hence, the induction hypothesis holds, and we may continue. Letting $A=\left\{a_{m}\right.$ : $m \in \omega\}$, we see that $(A, g)$ is a precanonical pair for $f \uparrow[V]^{2}$ (hence for $f$ ) such that $\operatorname{deg}(A \oplus g) \leq \operatorname{deg}(V)^{\prime} \leq \mathbf{a}$.

We are now in a position to give upper bounds on the Turing degrees of canonical sets for computable $f$. We prove the result in relativized form to facilitate the induction.

Theorem 4.5. Suppose that $n, p \geq 1, X \subseteq \omega, B \subseteq \omega$ is infinite, and $f:[B]^{n} \rightarrow$ $\omega \times p$. Suppose also that $B$ and $f$ are $X$-computable. If $n=1$, then there exists an $X$-computable set $C \subseteq B$ canonical for $f$. If $n \geq 2$ and $\mathbf{a} \gg \operatorname{deg}(X)^{(2 n-3)}$, there exists a set $C \subseteq B$ canonical for $f$ such that $\operatorname{deg}(C) \leq \mathbf{a}$.

Proof. We prove the theorem by induction on $n$. First, if $n=1$, notice that the set $C$ produced in the base case of the proof of Theorem 3.4 is $X$-computable if both $B$ and $f$ are. Suppose now that $n=2, B$ and $f:[B]^{2} \rightarrow \omega \times p$ are $X$-computable, and $\mathbf{a} \gg \operatorname{deg}(X)^{\prime}$. By Proposition 4.4 relativized to $X$, there exists a precanonical pair $(A, g)$ for $f$ with $\operatorname{deg}(A \oplus g) \leq \mathbf{a}$. By the inductive hypothesis, there exists a set $C$ canonical for $g:[A]^{1} \rightarrow \omega \times 2 p$ with $\operatorname{deg}(C) \leq \operatorname{deg}(A \oplus g) \leq \mathbf{a}$. By Claim 3.6, $C$ is canonical for $f$.

Suppose that $n \geq 2$ and the theorem holds for $n$. Suppose that both $B$ and $f:[B]^{n+1} \rightarrow \omega \times p$ are $X$-computable, and $\mathbf{a} \gg \operatorname{deg}(X)^{(2 n-1)}$. By Proposition 4.4 relativized to $X$, there exists a precanonical pair $(A, g)$ for $f$ with $A \oplus g \leq X^{\prime \prime}$. Applying the inductive hypothesis to $g:[A]^{n} \rightarrow \omega \times 2 p$, there exists $C \subseteq A$ canonical for $g:[A]^{n} \rightarrow \omega \times 2 p$ with $\operatorname{deg}(C) \leq \mathbf{a}$ since $\mathbf{a} \gg \operatorname{deg}(X)^{(2 n-1)}=\left(\operatorname{deg}(X)^{\prime \prime}\right)^{(2 n-3)} \geq$ $\operatorname{deg}(A \oplus g)^{(2 n-3)}$. By Claim 3.6, $C$ is canonical for $f$.

We immediately obtain bounds for the location of canonical sets in the arithmetical hierarchy. These bounds will be improved in the next section.

Corollary 4.6. Suppose that $n \geq 2, p \geq 1, B \subseteq \omega$ is infinite, and $f:[B]^{n} \rightarrow \omega \times p$. Suppose also that $B$ and $f$ are computable. There exists a $\Delta_{2 n-1}^{0}$ set $C \subseteq B$ canonical for $f$.

Proof. We know that $\mathbf{0}^{(2 n-2)} \gg \mathbf{0}^{(2 n-3)}$. Therefore, by Theorem 4.5, there exists a set $C \subseteq B$ canonical for $f$ such that $\operatorname{deg}(C) \leq \mathbf{0}^{(2 n-2)}$. Using Post's Theorem, we conclude that $C$ is $\Delta_{2 n-1}^{0}$.

The proof of Proposition 4.4 for the case $n=1$ relied on the ability to form a set of reasonably low complexity which either consists entirely of elements needing to be assigned finitary colors, or entirely of elements needing to be assigned infinitary colors. We next show that this special feature of $n=1$ is essential to finding precanonical pairs below any $\mathbf{a} \gg \mathbf{0}^{\prime}$. 
Theorem 4.7. There exists a computable $f:[\omega]^{3} \rightarrow \omega$ such that $\operatorname{deg}(A) \geq \mathbf{0}^{\prime \prime}$ whenever $(A, g)$ is a precanonical pair for $f$.

Proof. By Proposition 2.13, there exists a c.e. $h_{0}:[\omega]^{2} \rightarrow 2$ (that is, $\left\{x \in[\omega]^{2}\right.$ : $\left.h_{0}(x)=1\right\}$ is c.e.) such that for all sets $H$ homogeneous for $h_{0}$, we have $h_{0}\left([H]^{2}\right)=$ $\{0\}$ and $H \geq_{T} 0^{\prime}$. By the same result relative to $0^{\prime}$, there exists a $0^{\prime}$-c.e. $h_{1}:[\omega]^{2} \rightarrow 2$ (that is, $\left\{x \in[\omega]^{2}: h_{1}(x)=1\right\}$ is $0^{\prime}$-c.e.) such that for all sets $H$ homogeneous for $h_{1}$, we have $h_{1}\left([H]^{2}\right)=\{0\}$ and $H \oplus 0^{\prime} \geq_{T} 0^{\prime \prime}$.

Define $h:[\omega]^{2} \rightarrow 2$ by

$$
h(x)= \begin{cases}1 & \text { if either } h_{0}(x)=1 \text { or } h_{1}(x)=1, \\ 0 & \text { otherwise }\end{cases}
$$

Notice that $\left\{x \in[\omega]^{2}: h(x)=1\right\}$ is $0^{\prime}$-c.e. Suppose that $H$ is homogeneous for $h$. We then have $h\left([H]^{2}\right)=\{0\}$ because if $h\left([H]^{2}\right)=\{1\}$, an application of Ramsey's Theorem to the function $h_{2}:[H]^{2} \rightarrow 2$ given by

$$
h_{2}(x)= \begin{cases}0 & \text { if } h_{0}(x)=1, \\ 1 & \text { if } h_{0}(x)=0 \text { and } h_{1}(x)=1\end{cases}
$$

would give an infinite set $I$ such that either $h_{0}\left([I]^{2}\right)=\{1\}$ or $h_{1}\left([I]^{2}\right)=\{1\}$, a contradiction. Thus, $H$ is homogeneous for both $h_{0}$ and $h_{1}$. It follows that $H \geq_{T} 0^{\prime}$ and hence $H \geq_{T} H \oplus 0^{\prime} \geq_{T} 0^{\prime \prime}$.

Since $\left\{x \in[\omega]^{2}: h(x)=1\right\}$ is $0^{\prime}$-c.e., it is $\Sigma_{2}^{0}$, so there exists a computable $R(x, a, b)$ such that $h(x)=1 \leftrightarrow(\exists a)(\forall b) R(x, a, b)$ for all $x \in[\omega]^{2}$. Define $f:[\omega]^{3} \rightarrow$ $\omega$ as follows. Given $x \in[\omega]^{2}$ and $s \in \omega$ with $x<s$, let

$$
f(x, s)= \begin{cases}(\mu a<s)(\forall b<s) R(x, a, b) & \text { if }(\exists a<s)(\forall b<s) R(x, a, b), \\ s & \text { otherwise }\end{cases}
$$

(where $(\mu a)(\ldots)$ is the least $a$ such that $(\ldots))$. Notice that $f$ is computable. Furthermore, for all $x \in[\omega]^{2}$, we have $h(x)=1 \leftrightarrow \lim _{s} f(x, s)$ exists and is finite, and $h(x)=0 \leftrightarrow \lim _{s} f(x, s)=\infty$. Suppose that $(A, g)$ is a precanonical pair for $f$. For any $y \in[A]^{2}$, we either have $f\left(y, a_{1}\right)=f\left(y, a_{2}\right)$ for all $a_{1}, a_{2} \in A$ with $y<a_{1}<a_{2}$ (if $\pi_{2}(g(y))=0$ ), or $f\left(y, a_{1}\right) \neq f\left(y, a_{2}\right)$ for all $a_{1}, a_{2} \in A$ with $y<a_{1}<a_{2}$ (if $\pi_{2}(g(y))=1$ ). Therefore, given $y \in[A]^{2}$, if we let $a_{1}, a_{2} \in A$ be least such that $y<a_{1}<a_{2}$, we have $h(y)=1 \leftrightarrow f\left(y, a_{1}\right)=f\left(y, a_{2}\right)$ and $h(y)=0 \leftrightarrow f\left(y, a_{1}\right) \neq f\left(y, a_{2}\right)$. Hence, $h \uparrow[A]^{2}:[A]^{2} \rightarrow 2$ is $A$-computable. Since every set $H$ homogeneous for $h \uparrow[A]^{2}$ satisfies $h\left([H]^{2}\right)=\{0\}$, it follows from [9, Theorem 5.11] (relativized to $A$ ) that $h \uparrow[A]^{2}$, and hence $h$ itself, has an $A$ computable homogeneous set. Since every set homogeneous for $h$ has degree above $\mathbf{0}^{\prime \prime}$, we have $\operatorname{deg}(A) \geq \mathbf{0}^{\prime \prime}$.

Therefore, the bounds for canonical sets given by Theorem 4.5 are the best possible from an effective analysis of the above proof of the Canonical Ramsey Theorem. We show later that the bound given by Theorem 4.5 for exponent 2 is sharp.

\section{ARIthmetical Bounds}

Corollary 4.6 provided bounds in the arithmetical hierarchy for canonical sets when $B$ and $f:[B]^{n} \rightarrow \omega \times p$ were computable. In particular, we established that 
if $B$ is computable and $f:[B]^{2} \rightarrow \omega \times p$ is computable, then there exists a $\Delta_{3}^{0}$ set canonical for $f$. We first improve this result by showing that if $B$ is computable and $f:[B]^{2} \rightarrow \omega \times p$ is computable, then there exists a $\Pi_{2}^{0}$ set canonical for $f$.

Our proof of this result resembles in broad outline Jockusch's proof of Theorem 2.11, but requires significant care. We first outline the idea of the proof. For simplicity, assume that $f:[\omega]^{2} \rightarrow \omega$. Using a $0^{\prime}$-oracle, we enumerate the complement of a set $A$, which will be part of a precanonical pair for $f$. Instead of using an oracle to decide which color to assign to a new element, we blindly assign a color to a new element, hoping that the corresponding thinned set will be infinite, and continue. If we ever discover that the corresponding set is finite using a $0^{\prime}$-oracle, we change the color and discard all of the work performed after assigning the bad color.

As long as we proceed through the possible colors intelligently, this outline will work and will produce an infinite $\Pi_{2}^{0}$ set $A$ which is part of a precanonical pair. However, if we proceed through the colors naively, we may not be able to extract a $\Pi_{2}^{0}$ canonical set from $A$. For example, suppose that we first proceed through the finitely many possible infinitary colors (there are only finitely many because all infinitary colors distinct from the ones assigned to previous elements are equivalent), and then proceed through the finitary colors in increasing order. If at the end of this construction every color is assigned to only finitely many elements of $A$, it seems impossible to drop elements in the construction to thin out $A$ to a $\Pi_{2}^{0}$ canonical set. We want to drop elements that repeat earlier colors, but there does not seem to be a way to safely do this since the color at any given stage may change.

We thus carry out the construction in a slightly less intuitively natural manner which will allow us to extract a $\Pi_{2}^{0}$ canonical set. The idea is to first assign a new element a new infinitary color, then assign it infinitary colors already in use by previous elements in increasing order, then assign it new finitary colors in increasing order, and finally assign it finitary colors already in use by previous elements in increasing order. Of course, there are infinitely many new finitary colors at any stage, so we need a way to determine when to stop and move into used finitary colors. This can be done because the only reason why we reject all of the infinitary colors for a number $a$ is because the set $\{f(a, b): b \in Z\}$ (where $Z$ is the currently thinned out set we are working inside) is bounded (see Lemma 5.3 below), and we can find a bound using a $0^{\prime}$-oracle. Following this strategy, we will be able to extract a $\Pi_{2}^{0}$ canonical set from $A$. For example, if there are infinitely many distinct infinitary colors, we can perform the construction with the additional action of dropping any element from our final set if it ever needs to change color. This will result in a $\Pi_{2}^{0}\{0,1\}$-canonical set. On the other hand, if there are finitely many distinct infinitary colors, and an infinitary color $d$ which occurs infinitely often, then for the least such $d$ we can perform the construction, dropping any element from our final set if it ever needs to take on a finitary color or a used infinitary color greater than $d$. Modulo finitely many mistakes, this will result in a $\Pi_{2}^{0}\{1\}$-canonical set. The remaining cases are handled in a similar manner.

We now carry out the above sketch in the more general setting of a computable $f:[B]^{2} \rightarrow \omega \times p$ so that we can lift the result to higher exponents.

Theorem 5.1. Suppose that $p \geq 1, B \subseteq \omega$ is infinite, and $f:[B]^{2} \rightarrow \omega \times p$. Suppose also that $B$ and $f$ are computable. There exists a $\Pi_{2}^{0}$ set $C \subseteq B$ canonical for $f$. 
Proof. We first use a movable marker construction using a $0^{\prime}$-oracle to enumerate the complement of an infinite set $A=\left\{a_{0}<a_{1}<a_{2}<\ldots\right\} \subseteq B$. We denote by $a_{i}^{s}$ the position of the $(i+1)^{\text {st }}$ marker $\Lambda_{i}$ at the beginning of stage $s$. At the beginning of each stage $s$, we will have a number $n^{s}$ such that the markers currently having a position are exactly the $\Lambda_{i}$ for $i<n^{s}$, and for each $i<n^{s}$, we will have numbers $e_{i}^{s}$ (representing the current color for $a_{i}^{s}$ ) and $q_{i}^{s}$ (representing the current column of $\omega \times p$ for $a_{i}^{s}$ ) with $q_{i}^{s}<2 p$. Let $\beta^{s}$ be the greatest position of any marker up to stage $s\left(\beta^{s}=0\right.$ if $\left.s=0\right)$, and let $m^{s}=\max \left(\{0\} \cup\left\{\pi_{1}\left(f\left(b_{1}, b_{2}\right)\right): b_{1}<b_{2} \leq \beta^{s}\right\}\right)$. Given these and $k \leq n^{s}$, we say that a number $b$ is $k$-acceptable at $s$ if:

- $b \in B$.

- $b>\beta^{s}$.

- For all $i<k$ with $q_{i}^{s}<p, f\left(a_{i}^{s}, b\right)=\left(e_{i}^{s}, q_{i}^{s}\right)$.

- For all $i<k$ with $q_{i}^{s} \geq p, \pi_{2}\left(f\left(a_{i}^{s}, b\right)\right)=q_{i}^{s}-p$.

- For all $i<k$ with $q_{i}^{s} \geq p, \pi_{1}\left(f\left(a_{i}^{s}, b\right)\right)>m^{s}$.

- For all $i, j<k$ with $q_{i}^{s}=q_{j}^{s} \geq p, f\left(a_{i}^{s}, b\right)=f\left(a_{j}^{s}, b\right) \leftrightarrow e_{i}^{s}=e_{j}^{s}$.

Construction: First set $n^{0}=0$. Stage $s \geq 0$ : Assume inductively that we have $n^{s}$ such that the markers currently having a position are exactly the $\Lambda_{i}$ for $i<n^{s}$, along with $e_{i}^{s}$ and $q_{i}^{s}$ for all $i<n^{s}$. Enumerate into $\bar{A}$ all numbers $b \leq \beta^{s}$ such that $b \neq a_{i}^{s}$ for all $i<n^{s}$. Using a $0^{\prime}$-oracle, let $k^{s}$ be the largest $k \leq n^{s}$ such that there exists a number which is $k$-acceptable at $s$. Note that $k^{s}$ exists because every sufficiently large element of $B$ is 0 -acceptable at $s$. For each $q<2 p$, let $E_{q}^{s}=\left\{e_{i}^{s}: i<k^{s}\right.$ and $\left.q_{i}^{s}=q\right\}$.

Case 1: $k^{s}=n^{s}$ : Set $n^{s+1}=n^{s}+1$ and place marker $\Lambda_{n^{s}}$ on the least $k^{s}$ acceptable number. Leave all markers $\Lambda_{i}$ with $i<n^{s}$ in place, and let $e_{i}^{s+1}=e_{i}^{s}$ and $q_{i}^{s+1}=q_{i}^{s}$ for all $i<n^{s}$. Also, let $q_{n^{s}}^{s+1}=2 p-1$ and let $e_{n^{s}}^{s+1}=\min \left(\omega-E_{2 p-1}^{s}\right)$. (Place a new marker, and give it the first new infinitary color in the last column.)

Case 2: $k^{s}<n^{s}$ : Set $n^{s+1}=k^{s}+1$ and remove all markers $\Lambda_{i}$ with $k^{s}<i<n^{s}$. Leave all markers $\Lambda_{i}$ with $i \leq k^{s}$ in place and let $e_{i}^{s+1}=e_{i}^{s}$ and $q_{i}^{s+1}=q_{i}^{s}$ for all $i<k^{s}$. Let $a^{*}=a_{k^{s}}^{s}, e^{*}=e_{k^{s}}^{s}$ and $q^{*}=q_{k^{s}}^{s}$. We now have nine subcases to decide the values $e_{k^{s}}^{s+1}$ and $q_{k^{s}}^{s+1}$ : (Change a color, column, or both.)

Subcase 2.1: $q^{*} \geq p, E_{q^{*}}^{s} \neq \emptyset$ and $e^{*} \notin E_{q^{*}}^{s}$ : Let $q_{k^{s}}^{s+1}=q^{*}$ and $e_{k^{s}}^{s+1}=\min E_{q^{*}}^{s}$. (Take the first used infinitary color for this column.)

Subcase 2.2: $q^{*} \geq p, e^{*} \in E_{q^{*}}^{s}$, and $e^{*} \neq \max E_{q^{*}}^{s}$ : Let $q_{k^{s}}^{s+1}=q^{*}$ and $e_{k^{s}}^{s+1}=\min \left\{d \in E_{q^{*}}^{s}: d>e^{*}\right\}$. (Take the next used infinitary color for this column.)

Subcase 2.3: $q^{*} \geq p$ and either $E_{q^{*}}^{s}=\emptyset$ or $e^{*}=\max E_{q^{*}}^{s}$ : Let $q_{k^{s}}^{s+1}=q^{*}-1$ and $e_{k^{s}}^{s+1}=\min \left(\omega-E_{q^{*}-1}^{s}\right)$. (Move either to the next infinitary column, or move to the last finitary column, and assign the first unused color.)

Subcase 2.4: $q^{*}<p, e^{*} \notin E_{q^{*}}^{s}$, and there exists $b$ which is $k^{s}$-acceptable at $s$ with $f\left(a^{*}, b\right)>e^{*}$ : Let $q_{k^{s}}^{s+1}=q^{*}$ and $e_{k^{s}}^{s+1}=\min \left\{c \in \omega: c \notin E_{q^{*}}^{s}\right.$ and $\left.c>e^{*}\right\}$. (Take the next unused finitary color for this column.)

Subcase 2.5: $q^{*}<p, e^{*} \notin E_{q^{*}}^{s}, E_{q^{*}}^{s} \neq \emptyset$, and every $b$ which is $k^{s}$-acceptable at $s$ satisfies $f\left(a^{*}, b\right) \leq e^{*}$ : Let $q_{k^{s}}^{s+1}=q^{*}$ and $e_{k^{s}}^{s+1}=\min E_{q^{*}}^{s}$. (Take the first used finitary color for this column.)

Subcase 2.6: $q^{*}<p, e^{*} \in E_{q^{*}}^{s}$, and $e^{*} \neq \max E_{q^{*}}^{s}$ : Let $q_{k^{s}}^{s+1}=q^{*}$ and $e_{k^{s}}^{s+1}=\min \left\{c \in E_{q^{*}}^{s}: c>e^{*}\right\}$. (Move to the next used finitary color for this column.) 
Subcase 2.7: $0<q^{*}<p, E_{q^{*}}^{s}=\emptyset$, and every $b$ which is $k^{s}$-acceptable at $s$ satisfies $f\left(a^{*}, b\right) \leq e^{*}$ : Let $q_{k^{s}}^{s+1}=q^{*}-1$ and $e_{k^{s}}^{s+1}=\min \left(\omega-E_{q^{*}-1}^{s}\right)$. (Move to the next finitary column, and assign the first unused color.)

Subcase 2.8: $0<q^{*}<p$ and $e^{*}=\max E_{q^{*}}^{s}$ : Let $q_{k^{s}}^{s+1}=q^{*}-1$ and $e_{k^{s}}^{s+1}=$ $\min \left(\omega-E_{q^{*}-1}^{s}\right)$. (Move to the next finitary column, and assign the first unused color.)

Subcase 2.9: Otherwise: Let $q_{k^{s}}^{s+1}=0$ and $e_{k^{s}}^{s+1}=e^{*}+1$. (This case won't occur for any true element of $A$.)

\section{End Construction.}

Claim 5.2. For all $k \in \omega$, each $\operatorname{limit}_{\lim _{s}} a_{k}^{s}, \lim _{s} q_{k}^{s}$, and $\lim _{s} e_{k}^{s}$ exists, so we may define $a_{k}=\lim _{s} a_{k}^{s}, q_{k}=\lim _{s} q_{k}^{s}$, and $e_{k}=\lim _{s} e_{k}^{s}$.

Proof. We proceed by induction. We assume that the claim is true for all $i<k$ and prove it for $k$. Let $t$ be the least stage such that for all $i<k$ and all $s \geq t$, we have $a_{i}^{s}=a_{i}, q_{i}^{s}=q_{i}$, and $e_{i}^{s}=e_{i}$. At stage $t$, marker $\Lambda_{k}$ is placed on a number $b$ via Case 1 of the construction (since otherwise there exists $i<k$ such that either $q_{i}^{t+1} \neq q_{i}^{t}$ or $e_{i}^{t+1} \neq e_{i}^{t}$ ), so $n^{t+1}=k+1$. Since each of $a_{i}^{s}, q_{i}^{s}$, and $e_{i}^{s}$ for $i<k$ have come to their limits, we must have $k^{s} \geq k$ and hence $n^{s} \geq k+1$ for all $s>t$ by construction (because if $s>t$ is least such that $k^{s}<k$, then we enter Case 2, so one of $q_{k^{s}}^{s}$ or $e_{k^{s}}^{s}$ changes). Therefore, by construction, we never again move marker $\Lambda_{k}$, so $a_{k}^{s}=a_{k}^{t+1}$ for all $s \geq t+1$ and we may let $a_{k}=\lim _{s} a_{k}^{s}$.

We now show that $\lim _{s} q_{k}^{s}$ and $\lim _{s} e_{k}^{s}$ both exist by showing that $k^{s}=k$ for only finitely many $s>t$. This suffices, because $q_{k}^{s}$ and $e_{k}^{s}$ change only at such $s$. Suppose then that $k^{s}=k$ for infinitely many $s>t$. Let $Z=\{(d, q): p \leq q<2 p$ and $d \in E_{q}^{t} \cup\left\{\min \left(\omega-E_{q}^{t}\right)\right\}$. Following the construction through the first $|Z|$ many stages $s>t$ with $k^{s}=k$, we see that for all $(d, q) \in Z$, there is a unique $s_{(d, q)}>t$ such that $e_{k}^{s_{(d, q)}}=d, q_{k}^{s_{(d, q)}}=q$, and $k^{s_{(d, q)}}=k$. For each $(d, q) \in Z$, since $k^{s_{(d, q)}}=k$, there are no numbers which are $(k+1)$-acceptable at $s_{(d, q)}$. Let $r_{1}=\max \left\{s_{(d, q)}:(d, q) \in Z\right\}$. We need the following lemma.

Lemma 5.3. For all $s \geq r_{1}$, if $b$ is $k$-acceptable at $s$, then $\pi_{1}\left(f\left(a_{k}, b\right)\right) \leq m^{r_{1}}$.

Proof. Suppose that the lemma is false. Then there exists $s \geq r_{1}$ and a $b$ which is $k$-acceptable at $s$ such that $\pi_{1}\left(f\left(a_{k}, b\right)\right)>m^{r_{1}}$. Let $q=p+\pi_{2}\left(f\left(a_{k}, b\right)\right)$. For each $d$ with $(d, q) \in Z$, notice that $b$ is $k$-acceptable at $s_{(d, q)}$ (since $\left.t \leq s_{(d, q)} \leq r_{1} \leq s\right)$, but not $(k+1)$-acceptable at $s_{(d, q)}$. Therefore, for each $d$ with $(d, q) \in Z$, either $\pi_{1}\left(f\left(a_{k}, b\right)\right) \leq m^{s_{(d, q)}} \leq m^{r_{1}}$, or there exists $i<k$ with $q_{i}=q$ such that $f\left(a_{i}, b\right)=$ $f\left(a_{k}, b\right) \leftrightarrow e_{i} \neq d$. Since $\pi_{1}\left(f\left(a_{k}, b\right)\right)>m^{r_{1}}$, it follows that for all $d$ with $(d, q) \in Z$, there exists $i<k$ with $q_{i}=q$ such that $f\left(a_{i}, b\right)=f\left(a_{k}, b\right) \leftrightarrow e_{i} \neq d$. Letting $d=\min \left(\omega-E_{q}^{t}\right)$, we have $e_{i} \neq d$ for all $i<k$ with $q_{i}=q$, so we may choose $j<k$ with $q_{j}=q$ and $f\left(a_{j}, b\right)=f\left(a_{k}, b\right)$. Letting $d=e_{j}$, there exists $i<k$ with $q_{i}=q$ such that $f\left(a_{i}, b\right)=f\left(a_{k}, b\right) \leftrightarrow e_{i} \neq e_{j}$. Since $f\left(a_{j}, b\right)=f\left(a_{k}, b\right)$, this implies that $f\left(a_{i}, b\right)=f\left(a_{j}, b\right) \leftrightarrow e_{i} \neq e_{j}$, contrary to the fact that $b$ is $k$-acceptable at $s$. This is a contradiction, so the proof of the lemma is complete.

We now return to the proof of Claim 5.2. Notice that at stage $r_{1}$, we set $q_{k}^{r_{1}+1}=$ $q=p-1$, so $q_{k}^{s+1} \leq q_{k}^{s}<p$ for all $s>r_{1}$ by construction. Now, as we continue to follow the construction through stages $s$ with $k^{s}=k$, we must eventually reach a stage $s>r_{1}$ with $k^{s}=k$ such that we do not enter Subcase 2.4 (otherwise, we enter Subcase 2.4 infinitely often, so after $m^{r_{1}}$ such iterations, we reach an 
$s \geq r_{1}$ with $k^{s}=k$ and $e_{k}^{s} \geq m^{r_{1}}$ where every $b$ which is $k$-acceptable at $s$ satisfies $\pi_{1}\left(f\left(a_{k}, b\right)\right) \leq m^{r_{1}} \leq e_{k}^{s}$ by Lemma 5.3). Let $r_{2}$ be the least such stage. If $E_{q}^{t}=\emptyset$, then we either enter Subcase 2.7 and set $q_{k}^{r_{2}+1}=q-1$ (if $q>0$ ), or we enter Subcase 2.9 (if $q=0$ ). If $E_{q}^{t} \neq \emptyset$, then at stage $r_{2}$ we enter Subcase 2.5 and then repeatedly enter Subcase 2.6 whenever $k^{s}=k$ until we run through all elements of $E_{q}^{t}$, at which point we either enter Subcase 2.8 or Subcase 2.9. Therefore, in either case, we reach a stage $r_{3} \geq r_{2}$ where we either set $q_{k}^{r_{3}+1}=q-1$ or we enter Subcase 2.9. Now, the above argument works for the new value of $q$, so running through each $q$ with $q<p$ in reverse order, we see that we eventually reach a stage $r_{4}$ where we enter Subcase 2.9 .

Let $b$ be the least number which is $k$-acceptable at $r_{4}$ (such a number exists because otherwise we have $k^{r_{4}}<k$, which we know is not true). By construction, there exists a stage $s_{0} \leq r_{4}$ such that $e_{k}^{s_{0}}=\pi_{1}\left(f\left(a_{k}, b\right)\right), q_{k}^{s_{0}}=\pi_{2}\left(f\left(a_{k}, b\right)\right)$, and $k^{s_{0}}=k$. We then have that $b$ is $(k+1)$-acceptable at $s_{0}$, so $k^{s_{0}} \geq k+1$, a contradiction. It follows that there could not have been infinitely many $s>t$ with $k^{s}=k$, so the proof of the claim is complete.

Claim 5.4. Let $q<2 p$ be greatest such that $\left\{k: q_{k}=q\right\}$ is infinite.

(1) Suppose that $q \geq p$ and $\left\{e_{k}: q_{k}=q\right\}$ is infinite. Then $\left\{a_{k}: q_{k}=q\right.$ and $e_{k} \neq e_{i}$ for all $i<k$ with $\left.q_{i}=q\right\}$ is a $\Pi_{2}^{0}\{0,1\}$-canonical set for $f$.

(2) Suppose that (1) does not hold and $q \geq p$. Then there exists $d$ such that $\left\{k: q_{k}=q\right.$ and $\left.e_{k}=d\right\}$ is infinite, and for the least such $d$, the set $\left\{a_{k}: q_{k}=q\right.$ and $\left.e_{k}=d\right\}$ is a $\Pi_{2}^{0}\{1\}$-canonical set for $f$.

(3) Suppose that $q<p$ and $\left\{e_{k}: q_{k}=q\right\}$ is infinite. Then $\left\{a_{k}: q_{k}=q\right.$ and $e_{k} \neq e_{i}$ for all $i<k$ with $\left.q_{i}=q\right\}$ is a $\Pi_{2}^{0}\{0\}$-canonical set for $f$.

(4) Suppose that (3) does not hold, but $q<p$. Then there exists $c$ such that $\left\{k: q_{k}=q\right.$ and $\left.e_{k}=c\right\}$ is infinite, and for the least such $c$, the set $\left\{a_{k}: q_{k}=q\right.$ and $\left.e_{k}=c\right\}$ is a $\Pi_{2}^{0} \emptyset$-canonical set for $f$.

Proof. (1). Suppose that $q \geq p$ and $\left\{e_{k}: q_{k}=q\right\}$ is infinite. Let $C=\left\{a_{k}: q_{k}=q\right.$ and $e_{k} \neq e_{i}$ for all $i<k$ with $\left.q_{i}=q\right\}$. Notice that $C$ is infinite because $\left\{e_{k}: q_{k}=q\right\}$ is infinite. To see that $C$ is $\Pi_{2}^{0}$, perform the above construction, with the additional action of enumerating the number $a_{k^{s}}^{s}$ at stage $s$ if either

- $q_{k^{s}}^{s}<q$.

- $q_{k^{s}}^{s}=q$ and we enter Case 2 .

Then $a_{k}$ is not enumerated if and only if either

- $q_{k}>q$.

- $q_{k}=q$ and $e_{k} \neq e_{i}$ for all $i<k$ with $q_{i}=q$,

because at the first $s$ (if any) with $a_{k}^{s}=a_{k}$ and $q_{k}^{s}=q$, we set $e_{k}^{s}$ to a number different from $e_{i}$ for all $i<k$ with $q_{i}=q$, and entrance into Case 2 at any point will result either in $q_{k}<q$ or $e_{k}=e_{i}$ for some $i<k$ with $q_{i}=q$. Since $\left\{a_{k}: q_{k}>q\right\}$ is finite, $C$ is $\Pi_{2}^{0}$ (because removing finitely many elements from a $\Pi_{2}^{0}$ set leaves a $\Pi_{2}^{0}$ set). Suppose that $i<k, j<\ell, k \leq \ell$, and $a_{i}, a_{j}, a_{k}, a_{\ell} \in C$. Let $s$ be least such that $a_{\ell}^{s}=a_{\ell}$. If $k<\ell$, then $a_{\ell}$ is $(\max \{j, k\}+1)$-acceptable at $s$ by construction; hence $f\left(a_{j}, a_{\ell}\right)>m^{s} \geq f\left(a_{i}, a_{k}\right)$. If $k=\ell$ and $i \neq j$, then $a_{k}$ is $(\max \{i, j\}+1)$ acceptable at $s$; hence $f\left(a_{i}, a_{k}\right)=f\left(a_{j}, a_{k}\right) \leftrightarrow e_{i}=e_{j}$, so $f\left(a_{i}, a_{k}\right) \neq f\left(a_{j}, a_{k}\right)$ because $e_{i} \neq e_{j}$. Therefore, $f\left(a_{i}, a_{k}\right)=f\left(a_{j}, a_{\ell}\right) \leftrightarrow i=j$ and $k=\ell \leftrightarrow a_{i}=a_{j}$ and $a_{k}=a_{\ell}$. It follows that $C$ is a $\Pi_{2}^{0}\{0,1\}$-canonical set for $f$. 
(2). Suppose that (1) does not hold, i.e. $\left\{e_{k}: q_{k}=q\right\}$ is finite, and $q \geq p$. Let $d$ be least such that $\left\{k: q_{k}=q\right.$ and $\left.e_{k}=d\right\}$ is infinite, and let $C=\left\{a_{k}: q_{k}=q\right.$ and $\left.e_{k}=d\right\}$. To see that $C$ is $\Pi_{2}^{0}$, perform the above construction, with the additional action of enumerating the number $a_{k^{s}}^{s}$ at stage $s$ if either

- $q_{k^{s}}^{s}<q$.

- $q_{k^{s}}^{s}=q$ and we enter Subcase 2.2 and set $e_{k^{s}}^{s+1}$ to a number greater than $d$.

Then $a_{k}$ is not enumerated if and only if either

- $q_{k}>q$.

- $q_{k}=q$ and $e_{k} \neq e_{i}$ for all $i<k$ with $q_{i}=q$.

- $q_{k}=q$ and $e_{k} \leq d$,

because at the first $s$ (if any) with $a_{k}^{s}=a_{k}$ and $q_{k}^{s}=q$, we set $e_{k}^{s}$ to a number in $\omega \backslash\left\{e_{i}: i<k\right.$ and $\left.q_{i}=q\right\}$, after which the value of $e_{k}^{s}$ runs through the set $\left\{e_{i}: i<k\right.$ and $\left.q_{i}=q\right\}$ in increasing order until, if ever, we set $q_{k}^{s}<q$. Since $\left\{a_{k}: q_{k}>q\right\} \cup\left\{a_{k}: q_{k}=q, e_{k} \neq d\right.$, and $e_{k} \neq e_{i}$ for all $i<k$ with $\left.q_{i}=q\right\} \cup\left\{a_{k}\right.$ : $q_{k}=q$ and $\left.e_{k}<d\right\}$ is finite, it follows (by removing this finite set) that $C$ is $\Pi_{2}^{0}$. Suppose that $i<k, j<\ell, k \leq \ell$, and $a_{i}, a_{j}, a_{k}, a_{\ell} \in C$. Let $s$ be least such that $a_{\ell}^{s}=a_{\ell}$. If $k<\ell$, then $a_{\ell}$ is $(\max \{j, k\}+1)$-acceptable at $s$ by construction; hence $f\left(a_{j}, a_{\ell}\right)>m^{s} \geq f\left(a_{i}, a_{k}\right)$. If $k=\ell$, then $a_{k}$ is $(\max \{i, j\}+1)$-acceptable at $s$; hence $f\left(a_{i}, a_{k}\right)=f\left(a_{j}, a_{k}\right) \leftrightarrow e_{i}=e_{j}$, so $f\left(a_{i}, a_{k}\right)=f\left(a_{j}, a_{k}\right)$ because $e_{i}=d=e_{j}$. Therefore, $f\left(a_{i}, a_{k}\right)=f\left(a_{j}, a_{\ell}\right) \leftrightarrow k=\ell \leftrightarrow a_{k}=a_{\ell}$. It follows that $C$ is a $\Pi_{2}^{0}$ $\{1\}$-canonical set for $f$.

(3). Suppose that $q<p$ and $\left\{e_{k}: q_{k}=q\right\}$ is infinite. Let $C=\left\{a_{k}: q_{k}=q\right.$ and $e_{k} \neq e_{i}$ for all $i<k$ with $\left.q_{i}=q\right\}$. Notice that $C$ is infinite because $\left\{e_{k}: q_{k}=q\right\}$ is infinite. To see that $C$ is $\Pi_{2}^{0}$, perform the above construction, with the additional action of enumerating the number $a_{k^{s}}^{s}$ at stage $s$ if either

- $q_{k^{s}}^{s}<q$.

- $q_{k^{s}}^{s}=q$ and we enter Subcase 2.5.

Then $a_{k}$ is not enumerated if and only if either

- $q_{k}>q$.

- $q_{k}=q$ and $e_{k} \neq e_{i}$ for all $i<k$ with $q_{i}=q$,

because at the first $s$ (if any) with $a_{k}^{s}=a_{k}$ and $q_{k}^{s}=q$, we set $e_{k}^{s}$ to a number in $\omega \backslash\left\{e_{i}: i<k\right.$ and $\left.q_{i}=q\right\}$, and $e_{k}^{s}$ will continue to be an element of this set until we either enter into Subcase 2.5, at which point $e_{k}^{s}$ will never again be in this set, or we set $q_{k}^{s}<q$. Since $\left\{a_{k}: q_{k}>q\right\}$ is finite, it follows (by removing this finite set) that $C$ is $\Pi_{2}^{0}$. Suppose that $i<j$ and $a_{i}, a_{j} \in C$. Let $s$ be least such that $a_{j}^{s}=a_{j}$. By construction, $a_{j}$ is $(i+1)$-acceptable at $s$; hence $f\left(a_{i}, a_{j}\right)=$ $\left(e_{i}, q_{i}\right)=\left(e_{i}, q\right)$. Therefore, whenever $i<k, j<\ell$, and $a_{i}, a_{j}, a_{k}, a_{\ell} \in C$, we have $f\left(a_{i}, a_{k}\right)=f\left(a_{j}, a_{\ell}\right) \leftrightarrow\left(e_{i}, q\right)=\left(e_{j}, q\right) \leftrightarrow e_{i}=e_{j} \leftrightarrow i=j \leftrightarrow a_{i}=a_{j}$. It follows that $C$ is a $\Pi_{2}^{0}\{0\}$-canonical set for $f$.

(4). Suppose that (3) does not hold, i.e. $\left\{e_{k}: q_{k}=q\right\}$ is finite and $q<p$. Let $c$ be least such that $\left\{k: q_{k}=q\right.$ and $\left.e_{k}=c\right\}$ is infinite, and let $C=\left\{a_{k}: q_{k}=q\right.$ and $\left.e_{k}=c\right\}$. To see that $C$ is $\Pi_{2}^{0}$, perform the above construction, with the additional action of enumerating the number $a_{k^{s}}^{s}$ at stage $s$ if either

- $q_{k^{s}}^{s}<q$.

- $q_{k^{s}}^{s}=q$ and we enter Subcase 2.6 and set $e_{k^{s}}^{s+1}$ to a number greater than $c$. 
Then $a_{k}$ is not enumerated if and only if either

- $q_{k}>q$.

- $q_{k}=q$ and $e_{k} \neq e_{i}$ for all $i<k$ with $q_{i}=q$.

- $q_{k}=q$ and $e_{k} \leq c$,

because at the first $s$ (if any) with $a_{k}^{s}=a_{k}$ and $q_{k}^{s}=q$, we set $e_{k}^{s}$ to a number in $\omega \backslash\left\{e_{i}: i<k\right.$ and $\left.q_{i}=q\right\}$ and $e_{k}^{s}$ will continue to be an element of this set until, if ever, $e_{k}^{s}$ runs through the set $\left\{e_{i}: i<k\right.$ and $\left.q_{i}=q\right\}$ in increasing order until, if ever, we either set $e_{k}^{s}>c$ or we set $q_{k}^{s}<q$. Since $\left\{a_{k}: q_{k}>q\right\} \cup\left\{a_{k}: q_{k}=q, e_{k} \neq c\right.$, and $e_{k} \neq e_{i}$ for all $i<k$ with $\left.q_{i}=q\right\} \cup\left\{a_{k}: q_{k}=q\right.$ and $\left.e_{k}<c\right\}$ is finite, it follows (by removing this finite set) that $C$ is $\Pi_{2}^{0}$. Suppose that $i<j$ and $a_{i}, a_{j} \in C$. Let $s$ be least such that $a_{j}^{s}=a_{j}$. By construction, $a_{j}$ is $(i+1)$-acceptable at $s$; hence $f\left(a_{i}, a_{j}\right)=\left(e_{i}, q_{i}\right)=(c, q)$. Therefore, whenever $i<k, j<\ell$, and $a_{i}, a_{j}, a_{k}, a_{\ell} \in C$, we have $f\left(a_{i}, a_{k}\right)=(c, q)=f\left(a_{j}, a_{\ell}\right)$. It follows that $C$ is a $\Pi_{2}^{0} \emptyset$-canonical set for $f$.

Again, using a relativized version of the result for exponent 2 and induction, we can get bounds for higher exponents.

Theorem 5.5. Suppose that $n \geq 2, p \geq 1, X \subseteq \omega, B \subseteq \omega$ is infinite, and $f:[B]^{n} \rightarrow \omega \times p$. Suppose also that $B$ and $f$ are $X$-computable. There exists $a$ $\Pi_{2 n-2}^{0, X}$ set $C$ canonical for $f$.

Proof. We prove the theorem by induction on $n$. Theorem 5.1 relativized to $X$ gives the result for $n=2$. Suppose that the theorem holds for $n \geq 2$, and that $B$ and $f:[B]^{n+1} \rightarrow \omega \times p$ are $X$-computable. By Proposition 4.4 relativized to $X$, there exists a precanonical pair $(A, g)$ for $f$ with $A \oplus g \leq_{T} X^{\prime \prime}$. Applying the inductive hypothesis to $g:[A]^{n} \rightarrow \omega \times 2 p$, there exists $C \subseteq A$ canonical for $g$ such that $C$ is $\Pi_{2 n-2}^{0, X^{\prime \prime}}$. Notice that $C$ is $\Pi_{2 n}^{0, X}$. By Claim 3.6, $C$ is canonical for $f$.

Remark 5.6. By Claim 1.8, if $n \geq 1$ and $f:[\omega]^{n} \rightarrow 2$, then any set $C$ canonical for $f$ is homogeneous for $f$. Therefore, for each $n \geq 2$, there exists a computable $f:[\omega]^{n} \rightarrow 2$ with no $\Sigma_{n}^{0}$ set canonical for $f$ by Theorem 2.11. It follows that Theorem 5.5 gives a sharp bound in the arithmetical hierarchy for $n=2$.

\section{UPPER BOUNDS FOR MINHOMOGENEOUS SETS}

Although the Regressive Function Theorem follows immediately from the Canonical Ramsey Theorem, we can obtain better bounds on the Turing degrees and position in the arithmetical hierarchy of minhomogeneous sets for computable $f$ via a direct proof using $r$-cohesive sets. We follow the outline by defining preminhomogeneous pairs, proving their utility and existence, and then applying induction.

Definition 6.1. Suppose that $n \geq 1, B \subseteq \omega$ is infinite, and $f:[B]^{n+1} \rightarrow \omega$ is regressive. We call a pair $(A, g)$ where $A \subseteq B$ is infinite and $g:[A]^{n} \rightarrow \omega$, a preminhomogeneous pair for $f$ if for all $x \in[A]^{n}$ and all $a \in A$ with $x<a$, we have $f(x, a)=g(x)$.

Claim 6.2. Suppose that $n \geq 1, B \subseteq \omega$ is infinite, $f:[B]^{n+1} \rightarrow \omega$ is regressive, and $(A, g)$ is a preminhomogeneous pair for $f$. We then have that $g$ is regressive, and any $M \subseteq A$ minhomogeneous for $g$ is minhomogeneous for $f$. 
Proof. Given any $x \in[A]^{n}$, fix $a \in A$ with $x<a$ and notice that $g(x)=f(x, a)<$ $\min (x)$ if $\min (x)>0$, and $g(x)=f(x, a)=0$ if $\min (x)=0$, so $g$ is regressive. Suppose that $M \subseteq A$ is minhomogeneous for $g$. Fix $x_{1}, x_{2} \in[M]^{n}$ and $a_{1}, a_{2} \in M$ with $x_{1}<a_{1}, x_{2}<a_{2}$, and $\min \left(x_{1}, a_{1}\right)=\min \left(x_{2}, a_{2}\right)$. We then have $\min \left(x_{1}\right)=$ $\min \left(x_{2}\right)$; hence

$$
\begin{aligned}
f\left(x_{1}, a_{1}\right) & =g\left(x_{1}\right) & & (\text { since }(A, g) \text { is a preminhomogeneous pair for } f) \\
& =g\left(x_{2}\right) & & \left(\text { since } M \text { is homogeneous for } g \text { and } \min \left(x_{1}\right)=\min \left(x_{2}\right)\right) \\
& =f\left(x_{2}, a_{2}\right) & & (\text { since }(A, g) \text { is a prehomogeneous pair for } f) .
\end{aligned}
$$

Therefore, $M$ is minhomogeneous for $f$.

Proposition 6.3. Suppose that $n \geq 1, B \subseteq \omega$ is infinite, and $f:[B]^{n+1} \rightarrow \omega$ is regressive. Suppose also that $B$ and $f$ are computable and $\mathbf{a} \gg \mathbf{0}^{\prime}$. There exists a preminhomogeneous pair $(A, g)$ for $f$ such that $\operatorname{deg}(A \oplus g) \leq \mathbf{a}$. In particular, there exists a preminhomogeneous pair $(A, g)$ for $f$ such that $(A \oplus g)^{\prime} \leq_{T} 0^{\prime \prime}$.

Proof. By Theorem 2.19 and Lemma 4.3, we may fix an r-cohesive set $V \subseteq B$ such that $\operatorname{deg}(V)^{\prime} \leq \mathbf{a}$. Suppose that $x \in[B]^{n}$. We have $f(x, a) \leq \min (x)$ for all $a \in B$ with $x<a$, so the sets $Z_{c}=\{a \in B: x<a$ and $f(x, a)=c\}$ for $c$ with $0 \leq c \leq \min (x)$ are computable, pairwise disjoint, and have union $\{a \in B: x<a\}$. Since $V$ is r-cohesive, for each $c$ with $0 \leq c \leq \min (x)$, either $V \cap Z_{c}$ is finite or $V \cap \overline{Z_{c}}$ is finite. Therefore, there exists a unique $c_{x}$ with $0 \leq c_{x} \leq \min (x)$ such that $V \cap \overline{Z_{c_{x}}}$ is finite. Moreover, notice that the function from $[B]^{n}$ to $\omega$ given by $x \mapsto c_{x}$ is $V^{\prime}$-computable (since given $x \in[B]^{n}$, we can run through $b \in B$ in increasing order asking a $V^{\prime}$-oracle if all elements of $V$ greater than $b$ lie in a fixed $Z_{c}$ for some $c$ with $0 \leq c \leq \min (x)$ ).

We use a $V^{\prime}$-oracle to inductively construct a preminhomogeneous pair $(A, g)$ for $f$. Let $a_{0}, a_{1}, \ldots, a_{n-1}$ be the first $n$ elements of $V$. Suppose that $m \geq n-1$ and we have defined $a_{0}, a_{1}, \ldots, a_{m}$. Using a $V^{\prime}$-oracle, let $a_{m+1}$ be the least $b \in V$ such that $b>a_{m}$ and $f(x, b)=c_{x}$ for all $x \in\left[\left\{a_{i}: i \leq m\right\}\right]^{n}$ (notice that $a_{m+1}$ exists because $V \subseteq B$ is infinite and $f(x, b)=c_{x}$ for all sufficiently large $b \in V)$. Let $A=\left\{a_{m}: m \in \omega\right\}$ and define $g:[A]^{n} \rightarrow \omega$ by $g(x)=c_{x}$. Then $\operatorname{deg}(A \oplus g) \leq \operatorname{deg}(V)^{\prime} \leq \mathbf{a}$ and $(A, g)$ is a preminhomogeneous pair for $f$.

The last statement follows from the fact that there exists $\mathbf{a} \gg \mathbf{0}^{\prime}$ with $\mathbf{a}^{\prime} \leq \mathbf{0}^{\prime \prime}$ by relativizing the Low Basis Theorem to $\mathbf{0}^{\prime}$.

Remark 6.4. Proposition 6.3 can also be proved using an effective analysis of a proof using trees similar to the proof of Ramsey's Theorem using trees.

Theorem 6.5. Suppose that $n \geq 2, X \subseteq \omega, B \subseteq \omega$ is infinite, and $f:[B]^{n} \rightarrow \omega$ is regressive. Suppose also that $B$ and $f$ are $X$-computable and that $\mathbf{a} \gg \operatorname{deg}(X)^{(n-1)}$. There exists a set $M \subseteq B$ minhomogeneous for $f$ such that $\operatorname{deg}(M) \leq \mathbf{a}$.

Proof. We prove the theorem by induction on $n$. First, suppose that $n=2, B$ and $f:[B]^{2} \rightarrow \omega$ are $X$-computable, and $\mathbf{a} \gg \operatorname{deg}(X)^{\prime}$. By Proposition 6.3 relativized to $X$, there exists a preminhomogeneous pair $(A, g)$ for $f$ with $\operatorname{deg}(A \oplus g) \leq \mathbf{a}$. Since $A$ is trivially minhomogeneous for $g$, it follows from Claim 6.2 that $A$ is minhomogeneous for $f$.

Suppose that $n \geq 2$ and the theorem holds for $n$. Suppose that both $B$ and $f:[B]^{n+1} \rightarrow \omega$ are $X$-computable, and $\mathbf{a} \gg \operatorname{deg}(X)^{(n)}$. By Proposition 6.3 relativized to $X$, there exists a preminhomogeneous pair $(A, g)$ for $f$ with $(A \oplus g)^{\prime} \leq_{T}$ 
$X^{\prime \prime}$. Applying the inductive hypothesis to $g:[A]^{n} \rightarrow \omega$, there exists $M \subseteq A$ minhomogeneous for $g$ with $\operatorname{deg}(M) \leq \mathbf{a}$ since $\mathbf{a} \gg \operatorname{deg}(X)^{(n)}=\left(\operatorname{deg}(X)^{\prime \prime}\right)^{(n-2)} \geq$ $\left(\operatorname{deg}(A \oplus g)^{\prime}\right)^{(n-2)}=\operatorname{deg}(A \oplus g)^{(n-1)}$. By Claim 6.2, $M$ is minhomogeneous for $f$.

We can also use the above results to give bounds on the location of minhomogeneous sets in the arithmetical hierarchy.

Theorem 6.6. Suppose that $n \geq 2, X \subseteq \omega, B \subseteq \omega$ is infinite, and $f:[B]^{n} \rightarrow \omega$ is regressive. Suppose also that $B$ and $f$ are $X$-computable. There exists a $\Pi_{n}^{0, X}$ set minhomogeneous for $f$.

Proof. We prove the theorem by induction on $n$. Theorem 5.1 relativized to $X \subseteq \omega$ together with Claim 1.11 gives the result for $n=2$. Suppose that we know the theorem for $n \geq 2$, and that $B \subseteq \omega$ is infinite and $X$-computable, and $f:[B]^{n+1} \rightarrow$ $\omega$ is regressive and $X$-computable. By Proposition 6.3 relativized to $X$, there exists a precanonical pair $(A, g)$ for $f$ with $(A \oplus g)^{\prime} \leq_{T} X^{\prime \prime}$. Applying the inductive hypothesis to $g:[A]^{n} \rightarrow \omega$, there exists $M \subseteq A$ minhomogeneous for $g$ such that $M$ is $\Pi_{n}^{0, A \oplus g}$. Then $M$ is $\Pi_{n-1}^{0,(A \oplus g)^{\prime}}$, so it follows that $M$ is $\Pi_{n-1}^{0, X^{\prime \prime}}$, and hence $\Pi_{n+1}^{0, X}$. By Claim 6.2, $M$ is minhomogeneous for $f$.

Remark 6.7 . Theorem 6.6 in the case $n=2$ can also be proved without appealing to Theorem 5.1 by using a more natural generalization of the proof of Theorem 2.11 in the case $n=2$.

\section{LOWER BOUNDS FOR MINHOMOGENEOUS AND CANONICAL SETS}

We next turn our attention to lower bounds, aiming to show that the bounds given by Theorem 6.5 and Theorem 6.6 are sharp. For these purposes, it will be convenient to relax the definition of a regressive function.

Definition 7.1. Suppose that $n \geq 1, h: \omega \rightarrow \omega, B \subseteq \omega$ is infinite, and $f:[B]^{n} \rightarrow \omega$.

(1) We say that $f$ is $h$-regressive if for all $x \in[B]^{n}$, we have $f(x)<h(\min (x))$ whenever $h(\min (x))>0$, and $f(x)=0$ whenever $h(\min (x))=0$.

(2) A set $M$ is minhomogeneous for $f$ if $M \subseteq B, M$ is infinite, and for all $x, y \in[M]^{n}$ with $\min (x)=\min (y)$ we have $f(x)=f(y)$.

Remark 7.2. Notice that a function $f:[B]^{n} \rightarrow \omega$ is regressive if and only if it is $\iota$-regressive, where $\iota: \omega \rightarrow \omega$ is the identity function. ing.

By making very minor changes to the proof of Claim 1.11, we obtain the follow-

Claim 7.3. Suppose that $n \geq 1, h: \omega \rightarrow \omega, B \subseteq \omega$ is infinite, and $f:[B]^{n} \rightarrow \omega$ is $h$-regressive. If $C \subseteq B$ is canonical for $f$, then $C$ is minhomogeneous for $f$.

Therefore, by the Canonical Ramsey Theorem, every $h$-regressive function has a minhomogeneous set. Although $h$-regressive functions will be a convenient tool for us, their minhomogeneous sets provide no more complexity than those for regressive functions.

Proposition 7.4. Suppose that $n \geq 1, h: \omega \rightarrow \omega, B \subseteq \omega$ is infinite, and $f:[B]^{n} \rightarrow$ $\omega$ is h-regressive. Suppose also that $h, B$, and $f$ are computable. There exists a computable regressive $g:[B]^{n} \rightarrow \omega$ such that any set $M \subseteq B$ minhomogeneous for $g$ computes a minhomogeneous set for $f$. 
Proof. We may assume that $h$ is strictly increasing and never 0 (otherwise, replace $h$ by the function $h^{*}: \omega \rightarrow \omega$ defined by $h^{*}(0)=\max \{h(0), 1\}$ and $h^{*}(k+1)=$ $\max \left(\left\{h^{*}(k)+1, h(k+1)\right\}\right)$, and notice that $h^{*}$ is computable and that $f$ is $h^{*}$ regressive). Define $p: \omega \rightarrow \omega$ by letting $p(a)$ be the largest $b<a$ such that $h(b)<a$ if there exists a $b$ with $h(b)<a$, and letting $p(a)=0$ otherwise. Notice that $p$ is computable, increasing, and satisfies $\lim _{a} p(a)=\infty$.

Define $g:[B]^{n} \rightarrow \omega$ by setting

$$
g\left(a_{1}, \ldots, a_{n}\right)= \begin{cases}f\left(p\left(a_{1}\right), \ldots, p\left(a_{n}\right)\right)+1 & \text { if } 0<p\left(a_{1}\right)<\cdots<p\left(a_{n}\right), \\ 0 & \text { otherwise }\end{cases}
$$

If $g\left(a_{1}, \ldots, a_{n}\right) \neq 0$, then $0<p\left(a_{1}\right)<\cdots<p\left(a_{n}\right)$; hence

$$
\begin{aligned}
& g\left(a_{1}, \ldots, a_{n}\right)=f\left(p\left(a_{1}\right), \ldots, p\left(a_{n}\right)\right)+1 \\
& <h\left(p\left(a_{1}\right)\right)+1 \quad \text { (since } f \text { is } h \text {-regressive) } \\
& \leq a_{1} \text {, }
\end{aligned}
$$

so $g$ is regressive.

Suppose that $M \subseteq B$ is minhomogeneous for $g$. Suppose that $a_{1}, a_{1}^{\prime} \in M$ satisfy $a_{1}<a_{1}^{\prime}$ and $p\left(a_{1}\right)=p\left(a_{1}^{\prime}\right)>0$. Since $\lim _{a} p(a)=\infty$, there exist $a_{2}<a_{3}<\cdots<$ $a_{n} \in M$ such that $a_{1}^{\prime}<a_{2}$ and $0<p\left(a_{1}\right)=p\left(a_{1}^{\prime}\right)<p\left(a_{2}\right)<p\left(a_{3}\right)<\cdots<p\left(a_{n}\right)$. Since $M$ is minhomogeneous for $g$, we have

$$
\begin{aligned}
0 & =g\left(a_{1}, a_{1}^{\prime}, a_{3}, \ldots, a_{n}\right) \\
& =g\left(a_{1}, a_{2}, a_{3}, \ldots, a_{n}\right) \\
& =f\left(p\left(a_{1}\right), p\left(a_{2}\right), p\left(a_{3}\right), \ldots, p\left(a_{n}\right)\right)+1 \\
& \neq 0,
\end{aligned}
$$

a contradiction. Hence, if $a, b \in M$ satisfy $p(a)=p(b)>0$, then $a=b$.

Since $M$ is infinite, $p$ is increasing and computable, and $\lim _{a} p(a)=\infty$, it follows that the set $p(M)$ is infinite and $p(M) \leq_{T} M$. Suppose that $a_{1}<\cdots<a_{n}, b_{1}<$ $\cdots<b_{n} \in M$ with $0<p\left(a_{1}\right)<\cdots<p\left(a_{n}\right), 0<p\left(b_{1}\right)<\cdots<p\left(b_{n}\right)$ and $p\left(a_{1}\right)=p\left(b_{1}\right)$. Since $p\left(a_{1}\right)=p\left(b_{1}\right)>0$, we know from the above that $a_{1}=b_{1}$. Therefore, since $M$ is minhomogeneous for $g$, we have

$$
\begin{aligned}
f\left(p\left(a_{1}\right), \ldots, p\left(a_{n}\right)\right)+1 & =g\left(a_{1}, \ldots, a_{n}\right) \\
& =g\left(b_{1}, \ldots, b_{n}\right) \\
& =f\left(p\left(b_{1}\right), \ldots, p\left(b_{n}\right)\right)+1,
\end{aligned}
$$

so $f\left(p\left(a_{1}\right), \ldots, p\left(a_{n}\right)\right)=f\left(p\left(b_{1}\right), \ldots, p\left(b_{n}\right)\right)$. It follows that $p(M) \backslash\{0\}$ is a minhomogeneous set for $f$ which is $M$-computable.

Theorem 7.5. There is a computable regressive $f:[\omega]^{2} \rightarrow \omega$ such that $\operatorname{deg}(M) \gg$ $\mathbf{0}^{\prime}$ for every set $M$ which is minhomogeneous for $f$.

Proof. By Proposition 7.4, it suffices to find a computable $f:[\omega]^{2} \rightarrow \omega$ and a computable $h: \omega \rightarrow \omega$ such that $f$ is $h$-regressive and $\operatorname{deg}(M) \gg \mathbf{0}^{\prime}$ for every set $M$ which is minhomogeneous for $f$.

Let $K=\left\{e: \varphi_{e}(e) \downarrow\right\}$ be the usual computably enumerable halting set, and let $\left\{K_{s}\right\}_{s \in \omega}$ be a fixed computable enumeration of $K$. Let $\langle\cdot\rangle$ be a fixed effective 
bijection from $\omega^{<\omega}$ to $\omega$. Define $f_{1}: \omega^{2} \rightarrow 2$ by

$$
f_{1}(m, t)= \begin{cases}\varphi_{e, t}^{K_{t}}(n) & \text { if } m=\langle e, n\rangle \text { and } \varphi_{e, t}^{K_{t}}(n) \downarrow \in\{0,1\} \\ 0 & \text { otherwise. }\end{cases}
$$

Notice that $f_{1}$ is computable. Define a computable $f:[\omega]^{2} \rightarrow \omega$ as follows. Given $a, b \in \omega$ with $a<b$, let $f(a, b)=\left\langle f_{1}(0, b), f_{1}(1, b), \ldots, f_{1}(a, b)\right\rangle$. Notice that $f$ is $h$ regressive, where $h: \omega \rightarrow \omega$ is the computable function given by $h(k)=\max (\{0\} \cup$ $\left\{\left\langle a_{0}, a_{1}, \ldots, a_{k}\right\rangle+1: 0 \leq a_{i} \leq 1\right.$ for $\left.\left.i \leq k\right\}\right)$.

Suppose that $M$ is a minhomogeneous set for $f$. For each $e \in \omega$, define $g_{e}: \omega \rightarrow 2$ as follows. Given $n \in \omega$, find the least $a_{e, n}, b_{e, n} \in M$ with $\langle e, n\rangle \leq a_{e, n}<b_{e, n}$, and let $g_{e}(n)=f_{1}\left(\langle e, n\rangle, b_{e, n}\right)$. Notice that $g_{e}$ is $M$-computable for each $e \in \omega$.

Let $e, n \in \omega$. Since $M$ is minhomogeneous for $f$, we know that $f\left(a_{e, n}, b\right)=$ $f\left(a_{e, n}, b^{\prime}\right)$ for all $b, b^{\prime} \in M$ with $b, b^{\prime}>a_{e, n}$, so $f_{1}(\langle e, n\rangle, b)=f_{1}\left(\langle e, n\rangle, b^{\prime}\right)$ for all $b, b^{\prime} \in M$ with $b, b^{\prime}>a_{e, n}$. Thus, if $\varphi_{e}^{K}(n) \downarrow \in\{0,1\}$, then $g_{e}(n)=f_{1}\left(\langle e, n\rangle, b_{e, n}\right)=$ $\varphi_{e}^{K}(n)$ because $f_{1}(\langle e, n\rangle, t)=\varphi_{e}^{K}(n)$ for all sufficiently large $t \in \omega$.

Therefore, for all $e \in \omega$, if $\varphi_{e}^{K}$ is $\{0,1\}$-valued, then $g_{e}$ is a total $M$-computable extension. It follows that $M$ computes a total extension of every partial $\{0,1\}$ valued $\mathbf{0}^{\prime}$-computable function; hence $\operatorname{deg}(M) \gg \mathbf{0}^{\prime}$ by Proposition 2.3.

We can use the previous theorem to obtain lower bounds for exponents $n \geq 2$.

Theorem 7.6. For every $n \geq 2$ and $X \subseteq \omega$, there exists an $X$-computable regressive $f:[\omega]^{n} \rightarrow \omega$ such that $\operatorname{deg}(M \oplus X) \gg \operatorname{deg}(X)^{(n-1)}$ for every set $M$ minhomogeneous for $f$.

Proof. We prove the result by induction on $n$. The case $n=2$ follows by relativizing Theorem 7.5. Suppose that $n \geq 2$ and the result holds for $n$. Fix an $X^{\prime}$-computable regressive $g:[\omega]^{n} \rightarrow \omega$ such that $\operatorname{deg}\left(M \oplus X^{\prime}\right) \gg\left(\operatorname{deg}(X)^{\prime}\right)^{(n-1)}=\operatorname{deg}(X)^{(n)}$ for every set $M$ which is minhomogeneous for $g$. By the Limit Lemma, there exists an $X$-computable $g_{1}:[\omega]^{n+1} \rightarrow \omega$ such that $\lim _{a} g_{1}(x, a)=g(x)$ for all $x \in[\omega]^{n}$ and $g_{1}(y) \leq \min (y)$ for all $y \in[\omega]^{n+1}$. By Proposition 2.12 relativized to $X$ and the fact that $n+1 \geq 3$, there exists an $X$-computable $f_{1}:[\omega]^{n+1} \rightarrow 2$ such that for all infinite sets $H$ homogeneous for $f_{1}$, we have $f_{1}\left([H]^{n+1}\right)=\{0\}$ and $H \oplus X \geq_{T} X^{\prime}$. Define an $X$-computable $f:[\omega]^{n+1} \rightarrow \omega$ by

$$
f(y)= \begin{cases}0 & \text { if } f_{1}(y)=1 \\ g_{1}(y)+1 & \text { if } f_{1}(y)=0\end{cases}
$$

Notice that $f(y) \leq g_{1}(y)+1 \leq \min (y)+1<\min (y)+2$ for all $y \in[\omega]^{n+1}$; hence $f$ is $h$-regressive, where $h: \omega \rightarrow \omega$ is the computable function given by $h(k)=k+2$. By Proposition 7.4 relativized to $X$, it suffices to show that $\operatorname{deg}(M \oplus X) \gg \operatorname{deg}(X)^{(n)}$ for all sets $M$ minhomogeneous for $f$.

Suppose that $M$ is minhomogeneous for $f$. For each $a \in M$, let $c_{a}=f(a, x)$ for some (any) $x \in[M]^{n}$ with $a<x$. Let $Z=\left\{a \in M: c_{a}=0\right\}$. Since $f_{1}\left([Z]^{n+1}\right)=1$, it follows that $Z$ is finite. For any $a \in M \backslash Z$, we have $c_{a} \neq 0$; hence $f_{1}\left([M \backslash Z]^{n+1}\right)=0$ and $M \oplus X \equiv_{T}(M \backslash Z) \oplus X \geq_{T} X^{\prime}$. Furthermore, for any $x \in[M \backslash Z]^{n}$ and any $b \in M \backslash Z$ with $x<b$, we have $g_{1}(x, b)+1=f(x, b)=c_{\min (x)}$; hence $g(x)+1=c_{\min (x)}$ for all $x \in[M \backslash Z]^{n}$. It follows that $M \backslash Z$ is minhomogeneous for $g$; hence $\operatorname{deg}(M \oplus X) \geq \operatorname{deg}\left(M \oplus X^{\prime}\right) \gg \operatorname{deg}(X)^{(n)}$. 
As an immediate corollary of Theorem 7.6, we get the following corollary giving a lower bound for the position of minhomogeneous sets in the arithmetical hierarchy.

Corollary 7.7. For every $n \geq 2$, there exists a computable regressive $f:[\omega]^{n} \rightarrow \omega$ with no $\Sigma_{n}^{0}$ minhomogeneous set.

Proof. By Theorem 7.6 with $X=\emptyset$, there exists a computable regressive $f:[\omega]^{n} \rightarrow$ $\omega$ such that $\operatorname{deg}(M) \gg \mathbf{0}^{(n-1)}$ for every set $M$ minhomogeneous for $f$. Suppose that $M$ is a $\Sigma_{n}^{0}$ set minhomogeneous for $f$. Let $M_{1} \subseteq M$ be an infinite $\Delta_{n}^{0}$ subset of $M$, and notice that $M_{1}$ is minhomogeneous for $f$. Since $M_{1}$ is $\Delta_{n}^{0}$, it follows that $\operatorname{deg}\left(M_{1}\right) \leq \mathbf{0}^{(n-1)}$. Thus, it is not the case that $\operatorname{deg}\left(M_{1}\right) \gg \mathbf{0}^{(n-1)}$, a contradiction. Therefore, there is no $\Sigma_{n}^{0}$ set minhomogeneous for $f$.

Remark 7.8. Corollary 7.7 also follows from the corresponding result for Ramsey's Theorem (Theorem 2.11). Fix a computable $f:[\omega]^{n} \rightarrow 2$ such that no $\Sigma_{n}^{0}$ set is homogeneous for $f$. Define $f^{*}:[\omega]^{n} \rightarrow \omega$ by letting $f^{*}(x)=f(x)$ if $\min (x) \geq 2$ and $f^{*}(x)=0$ if $\min (x)<2$, and notice that $f^{*}$ is regressive. Suppose that $M^{*}$ is $\Sigma_{n}^{0}$ and minhomogeneous for $f^{*}$. Let $M$ be an infinite $\Delta_{n}^{0}$ subset of $M^{*}$ with $0,1 \notin M$, and notice that $M$ is also minhomogeneous for $f^{*}$. Define $g: M \rightarrow \omega$ by letting $g(a)=f^{*}(x)$ for some (any) $x \in[M]^{n}$ with $a=\min (x)$, and notice that $g \leq_{T} M$. If $M_{0}=\{a \in M: g(a)=0\}$ is infinite, then $M_{0}$ is homogeneous for $f$ and $M_{0}$ is $\Delta_{n}^{0}$ (since $M_{0} \leq_{T} M$ ), a contradiction. Otherwise, $M_{1}=\{a \in M: g(a)=1\}$ is infinite, so $M_{1}$ is homogeneous for $f$ and $M_{1}$ is $\Delta_{n}^{0}$ (since $M_{1} \leq_{T} M$ ), a contradiction. Therefore, there is no $\Sigma_{n}^{0}$ set minhomogeneous for $f^{*}$.

Corollary 7.9. For every $n \geq 2$, there exists a computable regressive $f:[\omega]^{n} \rightarrow \omega$ such that every $\Pi_{n}^{0}$ set $M$ minhomogeneous for $f$ satisfies $\operatorname{deg}(M) \geq \mathbf{0}^{(n)}$.

Proof. By Theorem 7.6 with $X=\emptyset$, there exists a computable regressive $f:[\omega]^{n} \rightarrow$ $\omega$ such that $\operatorname{deg}(M) \gg \mathbf{0}^{(n-1)}$ for every set $M$ minhomogeneous for $f$. If $M$ is a $\Pi_{n}^{0}$ set minhomogeneous for $f$, then $\operatorname{deg}(M) \gg \mathbf{0}^{(n-1)}$ and $\operatorname{deg}(M)$ is c.e. relative to $\mathbf{0}^{(n-1)}$; hence $\operatorname{deg}(M) \geq \mathbf{0}^{(n)}$ by the Arslanov Completeness Criterion.

Combining Theorem 6.5 and Theorem 7.6, we obtain the following corollary, analogous to Corollary 2.4.

Corollary 7.10. For every $n \geq 2$, there is a "universal" computable regressive $f:[\omega]^{n} \rightarrow \omega$, i.e. an $f$ such that given any set $M_{f}$ minhomogeneous for $f$ and any computable regressive $g:[\omega]^{n} \rightarrow \omega$, there exists a set $M_{g}$ minhomogeneous for $g$ such that $M_{g} \leq_{T} M_{f}$.

Using Claim 1.11, we can infer similar results for canonical sets for computable $f:[\omega]^{n} \rightarrow \omega$.

Corollary 7.11. For every $n \geq 2$, there exists a computable $f:[\omega]^{n} \rightarrow \omega$ such that $\operatorname{deg}(C) \gg \mathbf{0}^{(n-1)}$ for every set $C$ canonical for $f$.

The next corollary was discussed in Remark 5.6, but we also obtain it immediately from Corollary 7.7.

Corollary 7.12. For every $n \geq 2$, there exists a computable $f:[\omega]^{n} \rightarrow \omega$ such that no $\Sigma_{n}^{0}$ set is canonical for $f$.

Corollary 7.13. For every $n \geq 2$, there exists a computable $f:[\omega]^{n} \rightarrow \omega$ such that every $\Pi_{n}^{0}$ set $C$ canonical for $f$ satisfies $\operatorname{deg}(C) \geq \mathbf{0}^{(n)}$. 
Also, combining Theorem 4.5 and Corollary 7.11 for $n=2$, we get the following.

Corollary 7.14. There is a "universal" computable $f:[\omega]^{2} \rightarrow \omega$, i.e. an $f$ such that given any set $C_{f}$ canonical for $f$ and any computable $g:[\omega]^{2} \rightarrow \omega$, there exists a set $C_{g}$ canonical for $g$ such that $C_{g} \leq_{T} C_{f}$.

In contrast, it is shown in [13] that there does not exist a "universal" computable $f:[\omega]^{2} \rightarrow 2$ for Ramsey's Theorem.

In the previous chapter, we gave upper bounds for canonical sets for computable $f:[\omega]^{n} \rightarrow \omega$, in terms of both the Turing degrees and the arithmetical hierarchy. In this chapter, we provided lower bounds. These bounds give sharp characterizations when $n=2$, but the above upper bounds increase by two jumps for each successive value of $n$ while the lower bounds increase by only one for each successive value of $n$. In light of Theorem 4.7, I conjecture that the upper bounds provided in Theorem 4.5 and Theorem 5.5 are sharp.

Conjecture 7.15. For every $n \geq 3$, there exists a computable $f:[\omega]^{n} \rightarrow \omega$ such that $\operatorname{deg}(C) \gg \mathbf{0}^{(2 n-3)}$ for every set $C$ canonical for $f$.

Conjecture 7.16. For every $n \geq 3$, there exists a computable $f:[\omega]^{n} \rightarrow \omega$ such that no $\Sigma_{2 n-2}^{0}$ set is canonical for $f$.

\section{ReVERSE mathematical COROLlaries}

In this section, we discuss some straightforward reverse mathematical corollaries of the computability-theoretic analysis we've carried out thus far. We omit most of the details because some of the results appear in detail elsewhere, and the proofs given above translate in a straightforward manner to proofs from $\mathrm{RCA}_{0}$.

Definition 8.1. The following definitions are made in second-order arithmetic.

(1) $\mathrm{RT}_{p}^{n}$ is the statement that every $f:[\mathbb{N}]^{n} \rightarrow p$ has a homogeneous set.

(2) $\mathrm{RT}^{n}$ is the statement that for all $p \geq 1$, every $f:[\mathbb{N}]^{n} \rightarrow p$ has a homogeneous set.

(3) RT is the statement that for all $n, p \geq 1$, every $f:[\mathbb{N}]^{n} \rightarrow p$ has a homogeneous set.

(4) $\mathrm{CRT}^{n}$ is the statement that every $f:[\mathbb{N}]^{n} \rightarrow \mathbb{N}$ has a canonical set.

(5) CRT is the statement that for all $n \geq 1$, every $f:[\mathbb{N}]^{n} \rightarrow \mathbb{N}$ has a canonical set.

(6) $\mathrm{REG}^{n}$ is the statement that every regressive $f:[\mathbb{N}]^{n} \rightarrow \mathbb{N}$ has a minhomogeneous set.

(7) REG is the statement that for all $n \geq 1$, every regressive $f:[\mathbb{N}]^{n} \rightarrow \mathbb{N}$ has a minhomogeneous set.

(8) $\mathrm{ACA}_{0}^{\prime}$ is the statement that for all sets $Z$ and all $n$, the $n^{\text {th }}$ jump of $Z$ exists.

(9) $\mathrm{B} \Gamma$ (where $\Gamma$ is a set of formulas) is the statement of $\Gamma$-bounding; i.e. for any formula $\theta(a, b) \in \Gamma$ we have

$$
(\forall c)[(\forall a<c)(\exists b) \theta(a, b) \rightarrow(\exists m)(\forall a<c)(\exists b<m) \theta(a, b)] .
$$

Proposition 8.2. The following are equivalent over $\mathrm{RCA}_{0}$ :

(1) $\mathrm{ACA}_{0}$.

(2) $\mathrm{CRT}^{n}$ for any fixed $n \geq 2$.

(3) $\mathrm{REG}^{n}$ for any fixed $n \geq 2$. 
(4) $\mathrm{RT}^{n}$ for any fixed $n \geq 3$.

(5) $\mathrm{RT}_{p}^{n}$ for any fixed $n \geq 3$ and $p \geq 2$.

Proof. To see that (1) implies (2), examine the proof of Theorem 3.4 and notice that it can be formalized (in a completely straightforward manner) in $A_{C A}$. Since the proofs of Claim 1.11 and Claim 1.8 can be carried out in $\mathrm{RCA}_{0}$, it follows that (2) implies (3) and (4). Formalizing the proof of Theorem 7.5 in RCA $_{0}$ gives (3) implies (1). Clearly, (4) implies (5), and formalizing the proof of Proposition 2.12 in $\mathrm{RCA}_{0}$ gives (5) implies (1).

Remark 8.3. At the end of [12], Kanamori and McAloon state that the implication $\mathrm{REG}^{2} \rightarrow \mathrm{ACA}_{0}$ over $\mathrm{RCA}_{0}$ is due to Clote. Hirst (see [5, Theorem 6.14]), in his thesis, proved that the stronger statement "Every $h$-regressive $f:[\mathbb{N}]^{2} \rightarrow \mathbb{N}$ has a minhomogeneous set" implies $\mathrm{ACA}_{0}$ over RCA .

Proposition 8.4. The following are equivalent over $\mathrm{RCA}_{0}$ :

(1) $\mathrm{ACA}_{0}^{\prime}$,

(2) CRT,

(3) REG,

(4) RT.

Proof. To see that (1) implies (2), examine the proof of Theorem 3.4 and notice that it can be formalized for all exponents $n$ (in a completely straightforward manner) in $\mathrm{ACA}_{0}^{\prime}$. Since the proofs of Claim 1.11 and Claim 1.8 can be carried out in $\mathrm{RCA}_{0}$, it follows that (2) implies (3) and (4). Formalizing the proof of Theorem 7.6 in $\mathrm{RCA}_{0}$ gives (3) implies (1), and formalizing the proof of Proposition 2.12 in $\mathrm{RCA}_{0}$ gives (4) implies (1).

Proposition 8.5. The following are equivalent over $\mathrm{RCA}_{0}$ :

(1) $\mathrm{B} \Pi_{1}^{0}$,

(2) $\mathrm{B} \Sigma_{2}^{0}$,

(3) $\mathrm{RT}^{1}$,

(4) $\mathrm{CRT}^{1}$.

Proof. The equivalence of (1) and (2) is standard and can be found in [4, Lemma 2.10]. The equivalence of (1) and (3) is due to Hirst [5, Theorem 6.4], and can also be found in [2, Theorem 2.10]. Since the proof of Claim 1.8 can be carried out in $\mathrm{RCA}_{0}$, it follows that (4) implies (3).

We now show that (3) implies (4). Let $\mathfrak{M}$ be a model of $R C A_{0}+R^{1}$ and let $\mathbb{N}$ be the set of natural numbers in $\mathfrak{M}$. Suppose that $f: \mathbb{N} \rightarrow \mathbb{N}$ and $f \in \mathfrak{M}$. If there exists $p \in \mathbb{N}$ such that $f(n) \leq p$ for all $n \in \mathbb{N}$, then there exists a set $H \in \mathfrak{M}$ which is homogeneous for $f$ since $\mathrm{RT}_{p+1}^{1}$ holds in $\mathfrak{M}$, and such an $H$ is canonical for $f$. Suppose then that the range of $f$ is unbounded; i.e. for every $p \in \mathbb{N}$, there exists an $n \in \mathbb{N}$ with $f(n)>p$. Since $\mathfrak{M}$ satisfies $\Delta_{1}^{0}$ comprehension, we may recursively define a function $g \in \mathfrak{M}$ as follows. Let $g(0)=0$, and given $g(n)$, let $g(n+1)$ be the least $k \in \mathbb{N}$ such that $k>g(n)$ and $f(k)>f(g(n))$. Since $g$ is strictly increasing, and $g \in \mathfrak{M}$, it follows that $\operatorname{range}(g)$ is infinite and range $(g) \in \mathfrak{M}$. Notice that range $(g)$ is canonical for $f$. 


\section{Conclusion}

Putting together the characterizations of Turing degrees of solutions for computable instances of König's Lemma and the above partition theorems for exponent 2 , we see a close connection.

Summary 9.1. Let a be a Turing degree. The following are equivalent:

(1) $\mathbf{a} \gg \mathbf{0}^{\prime}$.

(2) For every computable $f:[\omega]^{2} \rightarrow 2$, there is a set $H$ homogeneous for $f$ such that $\operatorname{deg}(H)^{\prime} \leq \mathbf{a}$.

(3) For every computable regressive $f:[\omega]^{2} \rightarrow \omega$, there is a set $M$ minhomogeneous for $f$ such that $\operatorname{deg}(M) \leq \mathbf{a}$.

(4) For every computable $f:[\omega]^{2} \rightarrow \omega$, there is a set $C$ canonical for $f$ such that $\operatorname{deg}(C) \leq \mathbf{a}$.

For exponents $n \geq 3$, the Turing degrees characterizing the location of solutions for Ramsey's Theorem and the Regressive Function Theorem increase by one jump for each successive value of $n$, while our upper bounds for solutions for the Canonical Ramsey Theorem increase by two jumps for each successive value of $n$.

In terms of the arithmetical hierarchy, each of the above partition theorems for exponent 2 has $\Pi_{2}^{0}$ solutions for computable instances, but not necessarily $\Sigma_{2}^{0}$ solutions. For exponents $n \geq 3$ the location of solutions for Ramsey's Theorem and the Regressive Function Theorem increase by one jump for each successive value of $n$, while our upper bounds for solutions for the Canonical Ramsey Theorem increase by two jumps for each successive value of $n$.

Many open questions remain. A resolution of Conjecture 7.15 and Conjecture 7.16 is perhaps the most relevant to fill out the above web of connections between König's Lemma, Ramsey's Theorem, the Regressive Function Theorem, and the Canonical Ramsey Theorem. Furthermore, the following fundamental questions about the relationship between Ramsey's Theorem and König's Lemma remain open.

Question 9.2 (Seetapun). Does $\mathrm{RT}_{2}^{2}$ imply $\mathrm{WKL}_{0}$ over $\mathrm{RCA}_{0}$ ?

Other interesting open questions arise when we examine other partition theorems. One such theorem which seems closely related to the ones we've been discussing is the Thin Set Theorem.

Definition 9.3 (Friedman). Suppose that $n \geq 1, B \subseteq \omega$ is infinite, and $f:[B]^{n} \rightarrow$ $\omega$. We say that a set $T \subseteq B$ is thin for $f$ if $T$ is infinite and there exists $c \in \omega$ such that $f(x) \neq c$ for all $x \in[T]^{n}$.

Theorem 9.4 (Thin Set Theorem, Friedman). Suppose that $n \geq 1, B \subseteq \omega$ is infinite, and $f:[B]^{n} \rightarrow \omega$. There exists a set $T$ thin for $f$.

The Thin Set Theorem (for exponent $n$ ) is a simple consequence of Ramsey's Theorem (for exponent $n$ ). After Friedman's initial work, Cholak, Guisto, Hirst, and Jockusch [1] furthered the effective analysis of the Thin Set Theorem, and gave a tight characterization of the location of thin sets for computable $f:[\omega]^{n} \rightarrow \omega$ in the arithmetical hierarchy. However, little is known about the Turing degrees of such solutions or the reverse mathematical strengths of the principles themselves. For example, it is not known if it is possible to code any nontrivial information into the thin sets of a computable $f:[\omega]^{n} \rightarrow \omega$ for any $n$. 


\section{REFERENCES}

[1] Peter Cholak, Mariagnese Guisto, Jeffry Hirst, and Carl Jockusch, Jr., Free sets and reverse mathematics, Reverse Mathematics 2001 (Stephen G. Simpson, ed.), Lect. Notes Log. 21, Assoc. Symbol. Logic, La Jolla, CA, 2005. MR2185429 (2006g:03101)

[2] Peter A. Cholak, Carl G. Jockusch, and Theodore A. Slaman, On the strength of Ramsey's theorem for pairs, J. Symbolic Logic 66 (2001), no. 1, 1-55. MR1825173 (2002c:03094)

[3] P. Erdös and R. Rado, A combinatorial theorem, J. London Math. Soc. 25 (1950), 249-255. MR0037886 (12:322f)

[4] Petr Hájek and Pavel Pudlák, Metamathematics of first-order arithmetic, Perspectives in Mathematical Logic, Springer-Verlag, Berlin, 1993. MR1219738 (94d:03001)

[5] Jeffry L. Hirst, Combinatorics in subsystems of second order arithmetic, Ph.D. thesis, The Pennsylvania State University, 1987.

[6] Tamara J. Hummel and Carl G. Jockusch, Jr., Ramsey's theorem for computably enumerable colorings, J. Symbolic Logic 66 (2001), no. 2, 873-880. MR1833484 (2002f:03077)

[7] Carl Jockusch and Frank Stephan, A cohesive set which is not high, Math. Logic Quart. 39 (1993), no. 4, 515-530. MR1270396 (95d:03078)

[8] - Correction to: "A cohesive set which is not high" [Math. Logic Quart. 39 (1993), no. 4, 515-530], Math. Logic Quart. 43 (1997), no. 4, 569. MR1477624 (99a:03044)

[9] Carl G. Jockusch, Jr., Ramsey's theorem and recursion theory, J. Symbolic Logic 37 (1972), 268-280. MR0376319 (51:12495)

[10] Carl G. Jockusch, Jr. and Robert I. Soare, $\Pi_{1}^{0}$ classes and degrees of theories, Trans. Amer. Math. Soc. 173 (1972), 33-56. MR0316227 (47:4775)

[11] Akihiro Kanamori, The higher infinite, second ed., Springer Monographs in Mathematics, Springer-Verlag, Berlin, 2003, Large cardinals in set theory from their beginnings. MR1994835 (2004f:03092)

[12] Akihiro Kanamori and Kenneth McAloon, On Gödel incompleteness and finite combinatorics, Ann. Pure Appl. Logic 33 (1987), no. 1, 23-41. MR870685 (88i:03095)

[13] Joseph R. Mileti, Ramsey degrees, to appear.

[14] J. Paris and Leo A. Harrington, A mathematical incompleteness in Peano arithmetic, Handbook of Mathematical Logic (Jon Barwise, ed.), North-Holland Publishing Co., Amsterdam, 1977, pp. 1133-1142. MR0457132 (56:15351)

[15] Richard Rado, Note on canonical partitions, Bull. London Math. Soc. 18 (1986), no. 2, 123-126. MR818813 (87e:05013)

[16] F. P. Ramsey, On a problem in formal logic, Proc. London Math. Soc. (3) 30 (1930), 264-286.

[17] Dana Scott, Algebras of sets binumerable in complete extensions of arithmetic, Proc. Sympos. Pure Math., Vol. V, American Mathematical Society, Providence, R.I., 1962, pp. 117-121. MR0141595 (25:4993)

[18] David Seetapun and Theodore A. Slaman, On the strength of Ramsey's theorem, Notre Dame J. Formal Logic 36 (1995), no. 4, 570-582, Special Issue: Models of arithmetic. MR1368468 (96k:03136)

[19] Stephen G. Simpson, Degrees of unsolvability: a survey of results, Handbook of Mathematical Logic (Jon Barwise, ed.), North-Holland, Amsterdam, 1977, pp. 1133-1142. MR0457132 (56:15351)

[20] - Subsystems of second order arithmetic, Perspectives in Mathematical Logic, Springer-Verlag, Berlin, 1999. MR1723993 (2001i:03126)

[21] Robert I. Soare, Recursively enumerable sets and degrees, Perspectives in Mathematical Logic, Springer-Verlag, Berlin, 1987, A study of computable functions and computably generated sets. MR882921 (88m:03003)

[22] E. Specker, Ramsey's theorem does not hold in recursive set theory, Logic Colloquium '69 (Proc. Summer School and Colloq., Manchester, 1969), North-Holland, Amsterdam, 1971, pp. 439-442. MR0278941 (43:4667)

Department of Mathematics, University of Illinois, 1409 W. Green Street, Urbana, ILLINOIS 61801

Current address: Department of Mathematics, University of Chicago, 5734 S. University Ave.,

Chicago, Illinois 60637

E-mail address: mileti@math.uchicago.edu 